\title{
HEATING SOURCE LOCALIZATION IN A REDUCED TIME
}

\author{
SARA BEDdiAF ${ }^{a}$, LAURENT AUTRIQUE $^{a, *}$, LAETITIA PEREZ $^{b}$, JEAN-Claude JOLlY $^{a}$
}

\author{
${ }^{a}$ LARIS-ISTIA \\ University of Angers, 62 avenue Notre Dame du Lac, 49000 Angers, France \\ e-mail: laurent.autrique@univ-angers.fr \\ ${ }^{b}$ LTN-UMR 6607 \\ University of Nantes, rue Christian Pauc, BP50609, 44306 Nantes cedex 3, France \\ e-mail: laetitia.perez@univ-nantes.fr
}

\begin{abstract}
Inverse three-dimensional heat conduction problems devoted to heating source localization are ill posed. Identification can be performed using an iterative regularization method based on the conjugate gradient algorithm. Such a method is usually implemented off-line, taking into account observations (temperature measurements, for example). However, in a practical context, if the source has to be located as fast as possible (e.g., for diagnosis), the observation horizon has to be reduced. To this end, several configurations are detailed and effects of noisy observations are investigated.
\end{abstract}

Keywords: parameter identification, inverse heat conduction problem, optimal observation, source localization.

\section{Introduction}

In numerous engineering contexts, parameter identification is a crucial requirement when unknown parameters have to be determined in order to better understand or to control the occurring phenomena. As an illustration, let us mention, e.g., the identification of a thermal characteristic of a plasma in a nuclear fusion reactor (Mechhoud et al., 2013), the identification of boundary conditions (a temperature dependent heat transfer coefficient) in a plasma assisted chemical vapor deposition process (Rouquette et al., 2007a), the localization of weak heat sources in electronic devices (Rakotoniaina et al., 2002) or the electron beam welding process (Rouquette et al., 2007b). Among industrial applications, several usual objectives can be mentioned: predictive model validation, identification of systems dynamic behavior, estimation of thermo-physical properties, or diagnosis of processes or materials. In this paper, the proposed study is devoted to the localization of one or several stationary heating sources in a reduced time for a three-dimensional geometry. This non-linear problem can be formulated as an inverse heat conduction problem (IHCP) where the

* Corresponding author system thermal state is described by a set of partial differential equations (PDEs). Such inverse problems are ill posed in the sense of Hadamard (Alifanov et al., 1995; Isakov, 1998; Morozov, 1994). In numerous references, parameter estimation for heat flux identification is achieved considering numerical solution of inverse problems (Park et al., 1999; Zhou et al., 2010; Mohammadiun et al., 2011; Hasanov and Pektas, 2013). Implementation of an extended Kalman filter for online identification purposes related to the IHCP was proposed by Daouas and Radhouani (2004; 2007).

Such a technique, well known in signal processing, induces a time-lag on identified parameters and can be difficult to tune without a priori information (related to the noise distribution, for example). Configurations studied in both previous references are related to boundary heat flux identification considering a 1D mathematical model. In the works of Kolodziej et al. (2010) or Mierzwiczak and Kolodziej (2010; 2011; 2012), the method of fundamental solutions was successfully implemented for an IHCP. This meshless method is relatively new and is an attractive alternative to the finite or boundary element methods. In the work of Egger et al. (2009), recent investigations in a $3 \mathrm{D}$ domain were performed in order to identify a surface heat flux using 36 microthermocouples located 
quite closely underneath the investigated surface. A conjugate gradient method is employed for iterative criterion minimization and a weak formulation is based on the Galerkin finite-dimensional technique. A multilevel approach is mentioned in order to consider sequential identification for the acceleration of minimizing the Tikhonov functional (but there are no details related to numerical implementation or tests).

In the last decades, several methods have been successfully implemented in order to determine the location of a single point heating source or line heating sources in one or two-dimension geometry. For example, in the work of Silva Neto and Ozisik (1993), both a depth and heat flux of a single heating source were identified in a one-dimension geometry using the conjugate gradient algorithm. Then, in the work of Khachfe and Jarny (2000), heat fluxes and locations of two pointwise sources in a two-dimensional domain were identified.

Le Niliot and Lefèvre (2001) investigated identification of multiple steady line heat sources in a diffusive system and proposed its application in a 2D experimental configuration. Always focused on 2D geometry, Lefèvre and Le Niliot (2002) estimated the location and strength of line heat sources using an identification procedure based on a boundary integral formulation with transient fundamental solutions. In the work of Yi and Murio (2002), a source term identification in a 1D-IHCP is performed using a regularization procedure, based on the mollification method and a marching scheme for the solution of the stabilized problem.

Based on an earlier reference (Beck and Arnold, 1977) and using the boundary element method, parameter identification was successfully investigated (Le Niliot and Lefèvre, 2004) in order to solve an inverse problem of pointwise heat source identification. Recently, a two-step regularized inverse solution for two-dimensional heat source reconstruction was developed by Renault et al. (2008). Moreover, the authors proposed a spectral method for the estimation of a thermo-mechanical heat source (this method is based on the analytical solution of the direct problem in the Laplace-Fourier domain and applied to a two-dimensional problem of heat conduction) (Renault et al., 2010). Girault et al. (2010) implemented the modal identification method for the identification of both the location and the heat flux of a heating source in a two-dimensional heat diffusion problem.

In the following, the method proposed by Silva Neto and Ozisik (1993) as well as Khachfe and Jarny (2000) is extended to a three-dimensional geometry. The IHCP is solved in order to determine the locations of one or several stationary heating sources while assuming that heat fluxes are known. It is important to notice that the spatial distribution of heat sources is not pointwise and has to be taken into account in a small area of the domain surface. Source locations are determined given temperature evolutions observed by a few pointwise sensors. In this context and among various methods available to solve the IHCP, the iterative conjugate gradient method (CGM) has been implemented for the minimization of the quadratic cost function describing the error between the numerical model outputs and the sensor measurements (Hager and Zhang, 2006; Prudhomme and Nguyen, 1998). It is well known that for such iterative minimization the solution of three well posed problems (always in the sense of Hadamard) is required. The direct problem describing evolution of the system state (temperature) is solved in order to evaluate the quadratic cost function. The gradient of the cost function is obtained once the adjoint problem is solved. Then the sensitivity problem is considered for the determination of the step-length in a descent direction. As for noisy measurements, it is shown by Alifanov (1994) that the conjugate gradient method acts as a regularization method. In fact, a stop ping criterion (for the minimization algorithm) which takes into account noise standard deviation can be considered a regularization parameter. In the proposed study, localization in a reduced time is investigated in order to put in evidence the relation between noise levels, the number of unknown parameters and the duration of measurements.

This paper is organized as follows. In the next section, the investigated thermal system is exposed (the notation and the PDE system). In Section 3, the IHCP is formulated and a brief description of the conjugate gradient algorithm is proposed. In order to locate the heating sources, both the sensitivity and adjoint problems are presented. In Section 4, several numerical examples are provided for different situations: one, two, and three sources. A reduced time solution is investigated and the effect of noisy measurements is presented. Finally, conclusion and outlooks are briefly drawn up at the end of this paper.

\section{Direct problem}

This section is devoted to the description of thermal heating phenomena modeled by a set of partial differential equations. Let us consider a titanium square plate $\Omega \subset \mathbb{R}^{3}$ (length $L=5 \cdot 10^{-2} \mathrm{~m}$, thickness $e=10^{-3} \mathrm{~m}$ ); the domain boundary is denoted by $\partial \Omega \subset \mathbb{R}^{2}$ (see Fig. 1). The space variable is

$$
(x, y, z) \in]-\frac{L}{2}, \frac{L}{2}[\times]-\frac{e}{2}, \frac{e}{2}[\times]-\frac{L}{2}, \frac{L}{2}[
$$

and the time variable is $t \in T=\left[0 ; t_{f}\right]$ in seconds. The plate is heated on its lower face $\Gamma_{\text {heated }}=$ $\{(x,-e / 2, z) \in \partial \Omega\}$ by one or several heating sources $S_{j}\left(j=1, \ldots, N_{s}\right.$, where $N_{s}$ is the number of heating sources). For each source, the heat flux is denoted by $\phi_{j}$ 


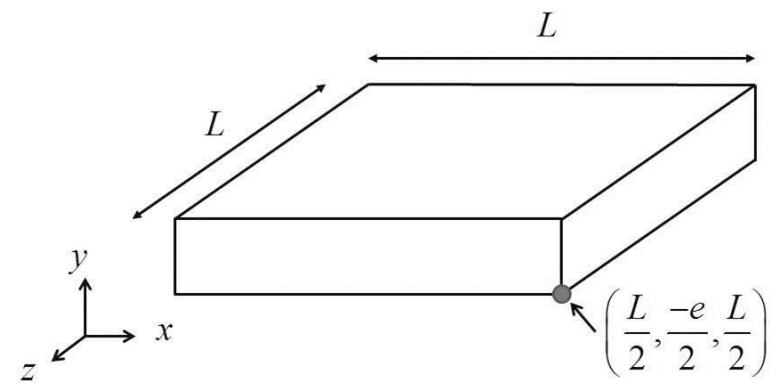

Fig. 1. Studied plate geometry.

and is not time dependent. Moreover, the heat flux $\phi_{j}$ is assumed to be uniform on the disk $D_{j} \in \Gamma_{\text {heated. Each }}$ disk (source) center is denoted by $I_{j}=\left(x_{j},-e / 2, z_{j}\right)$ and the disk radius is $r=0.002 \mathrm{~m}$. From a practical point of view, these circular heating flux spatial distributions could be obtained using a proper optical homogenizer. Such an optical device (also called the kaleidoscope) is commonly used to obtain a uniform flux (Chen et al., 1963) on a heated surface. Museux et al. (2012) used such equipment in order to obtain a uniform flux on a square for evaluation of burns. However, specific kaleidoscopes can be easily designed in order to obtain a circular distribution of a radiative heating flux on a plane target. Let us consider the following model for heating flux density in $W \cdot \mathrm{m}^{-2}$ :

$$
\Phi(x, z)=\sum_{j=1}^{N_{s}} \phi_{j} F_{j}(x, z),
$$

where

$$
F_{j}(\cdot)=\frac{1}{\pi}\left(-\operatorname{atan}\left(\mu\left(\sigma_{j}(x, z)-r\right)\right)+\frac{\pi}{2}\right),
$$

with $\mu \in \mathbb{R}^{+}$and

$$
\sigma_{j}(x, z)=\sqrt{\left(x-x_{j}\right)^{2}+\left(z-z_{j}\right)^{2}} .
$$

The parameter $\mu$ is chosen in order to describe the discontinuity in the neighborhood of the disk boundary. For the proposed configuration, $\mu=10^{5}$ is reasonable (image analysis is easily performed). It is important to notice that the parametrized function leads to a description of heat fluxes in a continuous and differentiable way.

Moreover, a natural convection phenomenon is considered on all plate boundaries $\partial \Omega$ : the convection coefficient is $h$ in $\mathrm{W} \cdot \mathrm{m}^{-2} \cdot \mathrm{K}^{-1}$. The $3 \mathrm{D}$ geometry considered is presented in Fig. 1. A more complex heat transfer and semi-transparent media are examined for predictive purposes in (Lormel et al., 2004). The temperature evolution is described by the following equations:

Direct problem $P_{\text {dir }}$ : Assuming a uniform initial temperature $\theta_{0}$ (equal to the ambient temperature) and the set of known parameters $P=\{\lambda, \rho c, h, \Phi, L, e\}$, determine the temperature $\theta(x, y, z ; t)$ as the solution of

$$
\left\{\begin{array}{l}
\forall(x, y, z ; t) \in \Omega \times T, \\
\lambda\left(\frac{\partial^{2} \theta}{\partial x^{2}}+\frac{\partial^{2} \theta}{\partial y^{2}}+\frac{\partial^{2} \theta}{\partial z^{2}}\right)=\rho c \frac{\partial \theta}{\partial t} \\
\forall(x, y, z) \in \Omega, \quad \theta(x, y, z ; 0)=\theta_{0} \\
\forall(x, y, z ; t) \in \partial \Omega \times T \\
-\lambda\left(\frac{\partial \theta}{\partial \vec{n}}\right)=h\left(\theta-\theta_{0}\right)-\Phi
\end{array}\right.
$$

where $\vec{n}$ is the outward normal unit vector.

Model input parameters are presented in Table 1. If all model inputs parameters are fixed, the previous direct problem described by (1) can be numerically solved using the finite element method (implemented, for example, in the Comsol-Multiphysics software interfaced with Matlab). In the following, the temperature $\theta(x, y, z ; t)$ numerically predicted at given spatial locations will be considered as the measured temperature for a set of sensors (see Section 3). In the proposed setting, the sensor spatial location is chosen as follows. It is crucial to select enough measurements points. It is obvious that a single sensor will be unable to find more than a single source (if heating fluxes are constant). The proposed configuration with three sensors is enough to allow triangulation. Since the heating source locations are unknown, in order to investigate the sample surface without a favored area, an equilateral triangle centered on the upper plate is meaningful. This is not a systematic procedure based on theoretical hypothesis about the investigated system a but experimental reasoning. If a large number of sensors is available, an optimal selection can be implemented based on the methodology developed by Perez and Vergnaud (2016). In the next section, the conjugate gradient method (CGM) is implemented to solve inverse problems in order to identify source locations $I_{j}$ in a reduced time.

\section{Inverse problem}

3.1. Formulation of the inverse heat conduction problem (IHCP). Assume that the coordinates of the source

Table 1. Notation and parameter definitions.

\begin{tabular}{|l||l|}
\hline Square length & $L=5 \cdot 10^{-2} \mathrm{~m}$ \\
Plate thickness & $e=2 \cdot 10^{-3} \mathrm{~m}$ \\
Initial temperature & $\theta_{0}=293 \mathrm{~K}$ \\
Heat flux & $\phi_{j}=10^{5} \mathrm{~W} \cdot \mathrm{m}^{-2}$ \\
Thermal conductivity & $\lambda=21.9 \mathrm{~W} \cdot \mathrm{m}^{-1} \cdot \mathrm{K}^{-1}$ \\
Volume heat & $\rho c=2.3510^{6} \mathrm{~J} \cdot \mathrm{m}^{-3} \cdot \mathrm{K}^{-1}$ \\
Natural convection coefficient & $h=20 \mathrm{~W} \cdot \mathrm{m}^{-2} \cdot \mathrm{K}^{-1}$ \\
\hline
\end{tabular}




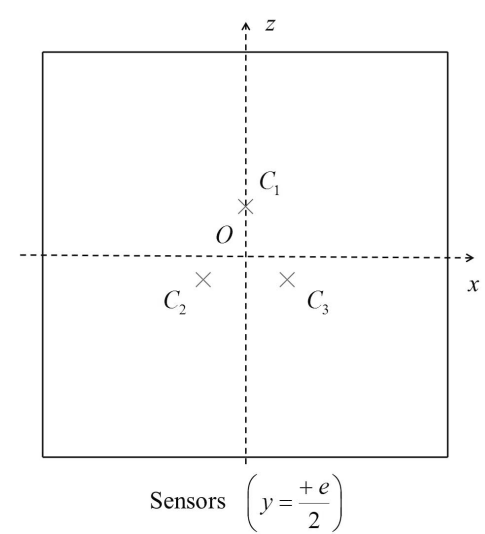

Fig. 2. Sensor location.

centers

$$
I_{j}^{\star}\left(x_{j}^{\star}, \frac{-e}{2}, z_{j}^{\star}\right)_{j=1, \ldots, N_{s}}
$$

are unknown. In the proposed setting, the aim of IHCP solution is to identify the source coordinates $\left(x_{j}^{\star}, z_{j}^{\star}\right)$ on the lower face given temperatures obtained from three sensors located on the opposite face $y=+e / 2$. This IHCP can be formulated as a classic optimization problem where a quadratic criterion denoted by $J(I)$ has to be minimized. Let us denote by $\hat{\theta}(t)$ the temperature measured at three sensors located on the upper face:

$$
\begin{gathered}
C_{1}\left(0, \frac{+e}{2}, 0.006\right), \\
C_{2}\left(-0.0052, \frac{+e}{2},-0.003\right), \\
C_{3}\left(0.0052, \frac{+e}{2},-0.003\right)
\end{gathered}
$$

(see Fig. 2). In the framework of optimal sensor location, several thermal situations encountered for high temperature processes are presented by Autrique et al. (2000; 2002). The quadratic criterion $J(I)$ is formulated as

$$
J(I)=\frac{1}{2} \int_{0}^{t_{f}} \sum_{i=1}^{3}\left(\theta\left(C_{i} ; t ; I\right)-\hat{\theta}_{i}(t)\right)^{2} \mathrm{~d} t,
$$

where $\theta\left(C_{i} ; t ; I\right)$ is the temperature predicted for sensor $i$ (given the source location $I$ ) while $\hat{\theta}_{i}(t)$ is the temperature measured at sensor $i$. The cost function is discretized using sampled measurements with a time step $\Delta t\left(N_{t}\right.$ measurements are available for each sensor):

$$
J_{d}(I)=\frac{\Delta t}{2} \sum_{n=1}^{N_{t}} \sum_{i=1}^{3}\left(\theta\left(C_{i} ; t_{n} ; I\right)-\hat{\theta}_{i}\left(t_{n}\right)\right)^{2} .
$$

The inverse problem is as follows:
Inverse problem $P_{\text {inv. }}$ : Find unknown source locations $I_{j=1, \ldots, N_{s}}^{\star}$ such that the quadratic functional $J(I)$ is minimum:

$$
I^{\star}=\arg \min _{\mathbb{R}^{2 N_{s}}} J(I),
$$

subject to the constraint $\theta(x, y, z ; t ; I)$ being a solution of the direct problem $P_{\text {dir }}$ (1).

In order to solve the previous ill-posed problem, the CGM algorithm is implemented (see, e.g., Alifanov, 1994; Silva Neto and Ozisik, 1993; Tarantola, 2005). It is important to note that the descent direction is reset to $\left(\beta^{k}=0\right)$ every $2 N_{s}$ iterations. Such a restart procedure for conjugate directions is discussed by Powell (1977). The direct problem (1) is discussed in Section 2. The following sections are concerned with the presentation of the sensitivity and adjoint problems related to Steps 3 and 4 of the CGM algorithm (see Algorithm 1).

3.2. Sensitivity problem. The formulation of this problem is helpful for the statement of the adjoint problem and for the estimation of the step-length in the descent direction. Let $\delta \theta(x, y, z ; t)$ be the temperature variation induced by a variation in the unknown parameters $\delta I=$ $\left\{\delta x_{j}, \delta y_{j}\right\}_{j=1, \ldots, N_{s}}$. Applications of variational calculus are presented by Weinstock (1952). For the PDE satisfied by the temperature $\theta(x, y, z ; t)+\epsilon \delta \theta(x, y, z ; t)$ (see the direct problem (1) with source locations $I+\epsilon \delta I)$, as $\epsilon \rightarrow 0$, the sensitivity problem (Khachfe and Jarny, 2000; 2001) becomes

Sensitivity problem $P_{\text {sens }}$ : Given the set of parameters $P=\{\lambda, \rho c, h, \delta \Phi, L, e\}$, determine $\delta \theta(x, y, z ; t)$ as the solution of

$$
\left\{\begin{array}{l}
\forall(x, y, z ; t) \in \Omega \times T, \\
\lambda\left(\frac{\partial^{2} \delta \theta}{\partial x^{2}}+\frac{\partial^{2} \delta \theta}{\partial y^{2}}+\frac{\partial^{2} \delta \theta}{\partial z^{2}}\right)=\rho c \frac{\partial \delta \theta}{\partial t}, \\
\forall(x, y, z) \in \Omega, \quad \delta \theta(x, y, z ; 0)=0, \\
\forall(x, y, z ; t) \in \partial \Omega \times T, \\
-\lambda\left(\frac{\partial \delta \theta}{\partial \vec{n}}\right)=h \delta \theta-\delta \Phi .
\end{array}\right.
$$

Here we have

$$
\delta \Phi(x, z)=\sum_{j=1}^{N_{s}} \phi_{j}\left(\left.\mathbf{1}\right|_{D_{j}^{+} \backslash D_{j}}-\left.\mathbf{1}\right|_{D_{j} \backslash D_{j}^{+}}\right)
$$

where

$$
\left.\mathbf{1}\right|_{A \backslash B}(x, z)= \begin{cases}1 & \text { if }(x, z) \in A \text { and }(x, z) \notin B \\ 0 & \text { otherwise, }\end{cases}
$$


and $D_{j}^{+}$is the disk with radius $r$ and heating source center $I_{j}+\epsilon \delta I_{j}$. This heating flux variation can be also formulated considering

$$
\delta \Phi(x, z)=\sum_{j=1}^{N_{s}} \phi_{j} \delta F_{j}(x, z)
$$

with

$$
\begin{aligned}
\delta F_{j}(x, z) & =\frac{1}{\pi} \frac{-\mu\left(\delta x_{j}\left(x_{j}-x\right)+\delta z_{j}\left(z_{j}-z\right)\right)}{\sigma_{j}(x, z)\left(1+\left(\mu\left(\sigma_{j}(x, z)-r\right)\right)^{2}\right)} \\
& =A_{j}(x, z) \delta x_{j}+B_{j}(x, z) \delta z_{j},
\end{aligned}
$$

where

$$
\begin{aligned}
& A_{j}(x, z)=\frac{1}{\pi} \frac{-\mu\left(x_{j}-x\right)}{\sigma_{j}(x, z)\left(1+\left(\mu\left(\sigma_{j}(x, z)-r\right)\right)^{2}\right)}, \\
& B_{j}(x, z)=\frac{1}{\pi} \frac{-\mu\left(z_{j}-z\right)}{\sigma_{j}(x, z)\left(1+\left(\mu\left(\sigma_{j}(x, z)-r\right)\right)^{2}\right)} .
\end{aligned}
$$

Finally, the heating flux variation is expressed as

$$
\delta \Phi(x, z)=\sum_{j=1}^{N_{s}} \phi_{j}\left(A_{j}(\cdot) \delta x_{j}+B_{j}(\cdot) \delta z_{j}\right) .
$$

3.3. Adjoint problem. This problem consists in determining the gradient of the cost function

$$
\overrightarrow{\nabla J^{k}}=\left(\frac{\partial J}{\partial x_{j}}, \frac{\partial J}{\partial z_{j}}\right)\left(x_{j}^{k}, z_{j}^{k}\right)_{j=1, \ldots, N_{s}}
$$

at each iteration $k$ of the CGM by introducing an adjoint function $\psi(x, y, z ; t)$. Other applications are presented by Jarny et al. (1991), Silva Neto and Ozisik (1993), Huang and Chen (1999), Khachfe and Jarny (2000; 2001), Rouquette et al. (2007a) and Perez et al. (2007).

Let us introduce the Lagrangian $\mathcal{L}(\theta, I, \psi)$ associated with the direct problem (1):

$$
\begin{aligned}
\mathcal{L}(\theta, I, \psi) & =J(\theta(\cdot)) \\
& +\int_{0}^{t_{f}} \int_{\Omega}\left(\rho c \frac{\partial \theta(\cdot)}{\partial t}-\lambda \Delta \theta(\cdot)\right) \psi(\cdot) \mathrm{d} t \mathrm{~d} \Omega .
\end{aligned}
$$

Let us consider

$$
\delta \mathcal{L}(\theta, I, \psi)=\frac{\partial \mathcal{L}}{\partial \theta} \delta \theta+\frac{\partial \mathcal{L}}{\partial I} \delta I+\frac{\partial \mathcal{L}}{\partial \psi} \delta \psi
$$

with

$$
\frac{\partial \mathcal{L}}{\partial I} \delta I=\sum_{j=1}^{N_{s}}\left(\frac{\partial \mathcal{L}}{\partial x_{j}} \delta x_{j}+\frac{\partial \mathcal{L}}{\partial z_{j}} \delta z_{j}\right) .
$$

Algorithm 1. CGM algorithm.

Step 1. Initialization $k=0: I_{j}^{0}\left(x_{j}^{0}, z_{j}^{0}\right)_{j=1, \ldots, N_{s}}$ is the initial unknown source center.

Step 2. Direct problem solution in order to calculate the temperature and the cost function $J\left(I^{k}\right)$.

- If $J\left(I^{k}\right) \leq J_{\text {stop }}$ (admissible threshold $J_{\text {stop }}$ will be discussed further), the minimization procedure is stopped and the current values $I_{j}^{k}\left(x_{j}^{k}, z_{j}^{k}\right)_{j=1, \ldots, N_{s}}$ are considered adequate estimates for source coordinates $I_{j}^{\star}$

- otherwise, the iterative procedure is continued.

Step 3. Adjoint problem solution in order to compute the functional gradient

$$
\overrightarrow{\nabla J^{k}}=\left(\frac{\partial J}{\partial x_{j}}, \frac{\partial J}{\partial z_{j}}\right)\left(x_{j}^{k}, z_{j}^{k}\right)
$$

for $j=1, \ldots, N_{s}$, and the next descent direction

$$
\overrightarrow{d^{k+1}}=-\overrightarrow{\nabla J^{k}}+\beta^{k} d^{k},
$$

where

$$
\beta^{k}=\frac{\left\|\overrightarrow{\nabla J^{k}}\right\|^{2}}{\left\|\overrightarrow{\nabla J^{k-1}}\right\|^{2}}
$$

$\left(\|\cdot\|\right.$ is the Euclidean norm and $\left.\beta^{0}=0\right)$.

Step 4. Sensitivity problem solution in order to estimate the step-length in the descent direction:

$$
\gamma^{k+1}=\arg \min _{\gamma \in \mathbb{R}}\left(I^{k}-\gamma \overrightarrow{d^{k+1}}\right)
$$

Step 5. Estimation of the new coordinates $I_{j}^{k+1}\left(x_{j}^{k+1}, z_{j}^{k+1}\right)$ using the following formulas:

$$
x_{j}^{k+1}=x_{j}^{k}-\gamma^{k+1}\left(\overrightarrow{d_{j}^{k+1}}\right)_{1}
$$

and

$$
z_{j}^{k+1}=z_{j}^{k}-\gamma^{k+1}\left(\overrightarrow{d_{j}^{k+1}}\right)_{2},
$$

where

$$
\left(\left(\overrightarrow{d_{j}^{k+1}}\right)_{1},\left(\overrightarrow{d_{j}^{k+1}}\right)_{2}\right)
$$

corresponds to the descent direction related to heat source $j$ and coordinates $(x, z)$.

Step 6. $k \leftarrow k+1$ and go to Step 2 . 
If $\theta$ is a solution to (1), then $\mathcal{L}(\theta, I, \psi)=J(I)$ and $\delta \mathcal{L}(\theta, I, \psi)=\delta J(\theta, I)$. If $\psi$ is fixed, then

$$
\frac{\partial \mathcal{L}}{\partial \psi} \delta \psi=0
$$

and $\delta \mathcal{L}(\theta, I, \psi)$ becomes

$$
\delta \mathcal{L}(\theta, I, \psi)=\frac{\partial \mathcal{L}}{\partial \theta} \delta \theta+\sum_{j=1}^{N_{s}}\left(\frac{\partial \mathcal{L}}{\partial x_{j}} \delta x_{j}+\frac{\partial \mathcal{L}}{\partial z_{j}} \delta z_{j}\right) .
$$

Then the choice of the fixed Lagrange multiplier $\psi(x, y, z ; t)$ is performed in order to satisfy the following equation:

$$
\frac{\partial \mathcal{L}}{\partial \theta} \delta \theta=0
$$

In order to determine $\psi(x, y, z ; t)$ which satisfies (7), while taking account of the previous equation (6), let us formulate $\delta \mathcal{L}(\theta, I, \psi)$ as follows:

$$
\begin{aligned}
\delta \mathcal{L}(\theta, I, \psi)= & \int_{0}^{t_{f}} \int_{\Omega} E \delta \theta \mathrm{d} t \mathrm{~d} \Omega \\
& +\int_{0}^{t_{f}} \int_{\Omega}\left(\rho c \frac{\partial \delta \theta}{\partial t}-\lambda \Delta \delta \theta\right) \psi \mathrm{d} t \mathrm{~d} \Omega,
\end{aligned}
$$

where

$$
\begin{aligned}
& E(x, y, z ; t) \\
& =\sum_{i=1}^{3}\left(\theta(x, y, z ; t)-\hat{\theta}_{i}(t)\right) \delta_{d}\left(x, y, z ; C_{i}\right)
\end{aligned}
$$

and $\delta_{d}\left(x, y, z ; C_{i}\right)$ is the Dirac distribution which is considered at the sensor location $C_{i}$. After several integrations by parts (versus space and time), while taking account of the sensitivity equations (4), Eqn. (7) is satisfied if the Lagrange multiplier $\psi$ is a solution to the following problem:

Adjoint problem $P_{\mathrm{adj}}$ : Assuming the set of parameters $P=\{\lambda, \rho c, h, L, e, E\}$, determine $\psi(x, y, z ; t)$ as a solution to

$$
\left\{\begin{array}{l}
\forall(x, y, z ; t) \in \Omega \times T, \\
\lambda\left(\frac{\partial^{2} \psi}{\partial x^{2}}+\frac{\partial^{2} \psi}{\partial y^{2}}+\frac{\partial^{2} \psi}{\partial z^{2}}\right)=E-\rho c \frac{\partial \psi}{\partial t} \\
\forall(x, y, z) \in \Omega, \quad \psi\left(x, y, z ; t_{f}\right)=0 \\
\forall(x, y, z ; t) \in \partial \Omega \times T, \quad-\lambda\left(\frac{\partial \psi}{\partial \vec{n}}\right)=h \psi
\end{array}\right.
$$

The corresponding derivation is presented in Appendix. If $\psi$ is a solution to the adjoint problem $P_{\text {adj }}$ (8) , then

$$
\delta \mathcal{L}(\theta, I, \psi)=-\int_{0}^{t_{f}} \int_{\partial \Omega} \psi \delta \Phi \mathrm{d} t \mathrm{~d}(\partial \Omega),
$$

with $\delta \Phi$ given in (5). Then $\delta \mathcal{L}(\theta, I, \psi)$ can be written as

$$
\begin{aligned}
\delta \mathcal{L}(\theta, I, \psi) & \\
= & -\int_{0}^{t_{f}} \int_{\partial \Omega} \psi \sum_{j=1}^{N_{s}} \frac{\phi_{j}(t)}{\pi}\left(A_{j}(\cdot) \delta x_{j}\right. \\
& \left.+B_{j}(\cdot) \delta z_{j}\right) \mathrm{d} t \mathrm{~d}(\partial \Omega) .
\end{aligned}
$$

Since

$$
\langle\overrightarrow{\nabla J}, \delta I\rangle_{L^{2}(T, \partial \Omega)}=\delta J=\delta \mathcal{L},
$$

the gradient of the cost function is

$$
\begin{aligned}
\overrightarrow{\nabla J}= & \left(\frac{\partial J}{\partial x_{j}}, \frac{\partial J}{\partial z_{j}}\right)_{j=1, \ldots, N_{s}} \\
= & \left(-\int_{0}^{t_{f}} \int_{\partial \Omega} \frac{\phi_{j}}{\pi} A_{j} \psi \mathrm{d} t \mathrm{~d}(\partial \Omega),\right. \\
& \left.-\int_{0}^{t_{f}} \int_{\partial \Omega} \frac{\phi_{j}}{\pi} B_{j} \psi \mathrm{d} t \mathrm{~d}(\partial \Omega)\right)_{j=1, \ldots, N_{s}} .
\end{aligned}
$$

3.4. Step-length in the descent direction. The step-length in the descent direction $\gamma^{k+1}$ corresponds to the optimal depth in the descent direction for the new value of the unknown heating source coordinates:

$$
\begin{aligned}
I^{k+1} & =I^{k}-\gamma^{k+1} \overrightarrow{d^{k+1}} \\
\gamma^{k+1} & =\arg \min _{\mathbb{R}} J\left(I^{k+1}\right),
\end{aligned}
$$

where

$$
\begin{aligned}
& J\left(I^{k+1}\right) \\
& =\frac{1}{2} \int_{0}^{t_{f}} \sum_{i=1}^{3}\left(\theta\left(C_{i} ; t ; I^{k}-\gamma^{k+1} \overrightarrow{d^{k+1}}\right)-\hat{\theta}_{i}(t)\right)^{2} \mathrm{~d} t .
\end{aligned}
$$

For small variations in heating source localizations, the problem can be linearized and the temperature is written as

$$
\begin{aligned}
& \theta\left(C_{i} ; t ; I^{k}-\gamma^{k+1} \overrightarrow{d^{k+1}}\right) \\
& \quad=\theta\left(C_{i} ; t ; I^{k}\right)-\gamma^{k+1} \delta \theta \overrightarrow{d^{k+1}}\left(C_{i} ; t\right) .
\end{aligned}
$$

Since $\delta \theta \underset{d^{k+1}}{\longrightarrow}\left(C_{i} ; t\right)$ is the sensitivity function induced by the variation in the heating source coordinates denoted by $\overrightarrow{d^{k+1}}$, we have

$$
\begin{aligned}
& J\left(I^{k}-\gamma^{k+1} \overrightarrow{d^{k+1}}\right) \\
& =\frac{1}{2} \int_{0}^{t_{f}} \sum_{i=1}^{3}\left(\theta\left(C_{i} ; t ; I^{k}\right)-\hat{\theta}_{i}(t)\right)^{2} \mathrm{~d} t \\
& \quad+\frac{\left(\gamma^{k+1}\right)^{2}}{2} \int_{0}^{t_{f}} \sum_{i=1}^{3}\left(\delta \theta \overrightarrow{d^{k+1}}\left(C_{i} ; t\right)\right)^{2} \mathrm{~d} t
\end{aligned}
$$




$$
\begin{aligned}
& -\gamma^{k+1} \int_{0}^{t_{f}} \sum_{i=1}^{3}\left(\theta\left(C_{i} ; t ; I^{k}\right)-\hat{\theta}_{i}(t)\right) \\
& \times \delta \theta \underset{d^{k+1}}{\longrightarrow}\left(C_{i} ; t\right) \mathrm{d} t .
\end{aligned}
$$

In order to minimize the quadratic functional $J\left(I^{k}-\gamma^{k+1} \overrightarrow{d^{k+1}}\right), \gamma^{k+1}$ is determined such that

$$
\frac{\partial J\left(I^{k}-\gamma^{k+1} \overrightarrow{d^{k+1}}\right)}{\partial \gamma}=0
$$

and then

$$
\begin{aligned}
& \frac{\partial J\left(I^{k}-\gamma^{k+1} \overrightarrow{d^{k+1}}\right)}{\partial \gamma} \\
& =\gamma^{k+1} \int_{0}^{t_{f}} \sum_{i=1}^{3}\left(\delta \theta \overrightarrow{d^{k+1}}\left(C_{i} ; t\right)\right)^{2} \mathrm{~d} t \\
& \left.\quad-\int_{0}^{t_{f}} \sum_{i=1}^{3}\left(\theta\left(C_{i} ; t ; I^{k}\right)-\hat{\theta}_{i}(t)\right)\right) \delta \theta \overrightarrow{d^{k+1}}\left(C_{i} ; t\right) \mathrm{d} t .
\end{aligned}
$$

Then the step-length in the descent direction $\gamma^{k+1}$ is

$$
=\frac{\left.\int_{0}^{t_{f}} \sum_{i=1}^{3}\left(\theta\left(C_{i} ; t ; I^{k}\right)-\hat{\theta}_{i}(t)\right)\right) \delta \theta \overrightarrow{d^{k+1}}(\cdot) \mathrm{d} t}{\int_{0}^{t_{f}} \sum_{i=1}^{3}\left(\delta \theta \overrightarrow{d^{k+1}}(\cdot)\right)^{2} \mathrm{~d} t} .
$$

At each iteration $k$, the sensitivity problem $P_{\text {sens }}$ (4) has to be solved in the descent direction $\overrightarrow{d^{k+1}}$ to compute the step-length in the descent direction $\gamma^{k+1}$.

3.5. Admissible level of minimization. In order to implement the algorithm of the CGM, a threshold $J_{\text {stop }}$ has to be defined in order to stop the iterative minimization procedure. If all perturbations can be neglected and the experiment takes place in ideal conditions, then, without noisy measurements and model errors, $J_{\text {stop }}$ can be ideally fixed close to zero. In this numerical study, observations are obtained from a given direct problem (where heat source locations

$$
I_{j}^{\star}\left(x_{j}^{\star}, \frac{-e}{2}, z_{j}^{\star}\right)_{j=1, \ldots, N_{s}}
$$

are assumed to be known). These simulated observations are taken into account to determine the source locations in a reduced time. If temperature measurements are not arbitrarily disturbed, the minimization algorithm is stopped when $J\left(I^{k}, \theta\right)<J_{\text {stop }}=10^{-3} \mathrm{~K}^{2} \cdot \mathrm{s}$. For such a value, source locations are well estimated since the additional criterion (tracking error)

$$
\operatorname{TE}\left(I^{k}\right)=\sum_{j=1}^{N_{s}} \sqrt{\left(x_{j}^{k}-x_{j}^{\star}\right)^{2}+\left(z_{j}^{k}-z_{j}^{\star}\right)^{2}}
$$

is quite small, where $I_{j}^{k}\left(x_{j}^{k},-e / 2, z_{j}^{k}\right)$ is the estimated location of the heat source $I_{j}^{\star}\left(x_{j}^{\star},-e / 2, z_{j}^{\star}\right)$ at iteration $k$. In the numerical setting considered, where $I_{j}^{\star}$ are known, TE is relevant information to investigate the algorithm behavior. The tracking error is considered a stopping criterion and the algorithm is stopped if $\mathrm{TE}\left(I^{k}\right)<10^{-3} \mathrm{~m}$. It is important to notice that, for realistic experimental situations when heat source locations are unknown, the tracking error cannot be calculated. Then the stopping criterion can be considered equal to

$$
J_{\text {stop }}=\Delta t \frac{N_{c}}{2} \sigma^{2} N_{t},
$$

where $\sigma$ is the standard deviation of the Gaussian noise and $N_{t}$ is the number of observations for each of $N_{c}=3$ sensors. Such a stopping strategy is discussed by Alifanov (1994).

\section{Numerical results}

In the following paragraphs, several configurations are investigated in order to illustrate CGM implementation and its regularization effect in the context of identification of heat source locations. For one, two or three heat sources, the minimization of the cost function and the evolution of the tracking error are shown for observations obtained at three sensors (with or without noise). Numerical results are obtained using the Comsol-Multiphysics solver interfaced with Matlab software with/without noisy measurements, for different values of the final time $t_{f}$ (this key parameter can also be considered the necessary measurement time).

4.1. Case A.1. In this first case, the plate is heated with a single source located at $I(0.01,-e / 2,0.01)$ in meters (see Fig. 3). Assume that $t_{f}=1 \mathrm{~s}$. Numerical results for the direct problem are presented in Fig. 4. Observations are performed every $0.1 \mathrm{~s}$ and the temperature values numerically obtained are considered as measurements $\theta\left(C_{i}, t\right)$ for the set of sensors $C_{i}$ (see Fig. 2). In the following, the source coordinates are unknown. In order to identify these unknown coordinates, the IHCP described by $P_{\text {inv }}$ (see Section 3 ) is solved. The initial value of source localization is given by $I(0.01,-e / 2,0)$. The solution of $P_{\text {inv }}$ is performed using CGM and results are presented hereafter. The algorithm is stopped for $J\left(I^{k}, \theta\right)<J_{\text {stop }}=10^{-3}$. In Fig. 5, it is shown that after eight iterations, criterion $J\left(I^{k}, \theta\right)$ converges under the adopted stopping criterion. Moreover, evolution of the tracking error, which decreases at each iteration, confirms the correct behavior of the minimization algorithm. For $J\left(I^{k}, \theta\right)<J_{\text {stop }}=10^{-3}$, the tracking error is lower than $10^{-3} \mathrm{~m}$. Coordinate evolution (versus iteration $k$ ) of the sought source is shown in Fig. 6 The identified 


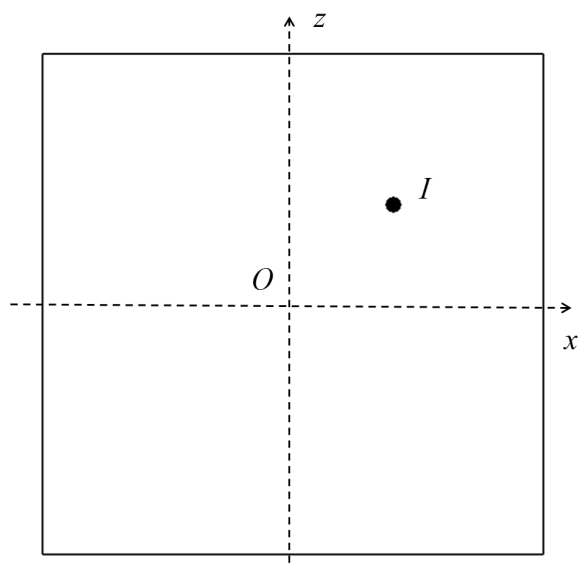

Fig. 3. Source location $y=-e / 2$ : Case A.1.

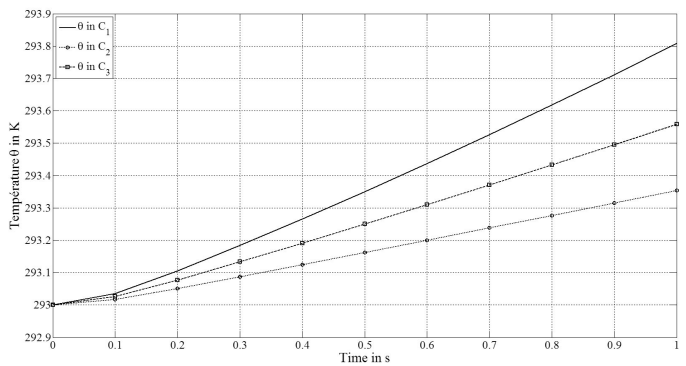

Fig. 4. Direct problem solution: Case A.1.

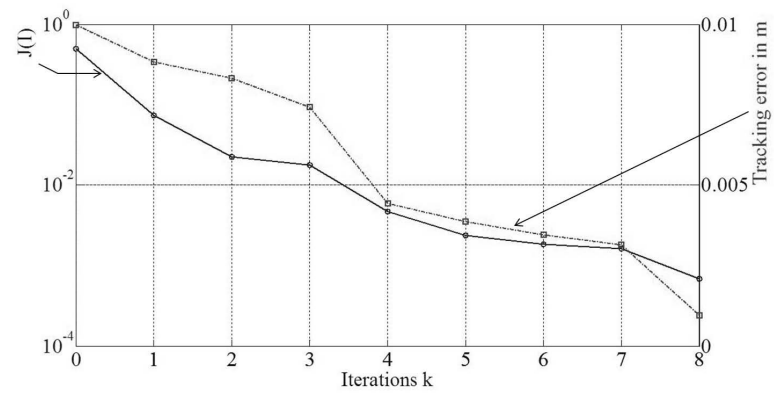

Fig. 5. Cost function and the tracking error: Case A.1.

Table 2. Criterion values vs. iteration $k$ : Case A.1.

\begin{tabular}{|c||ccccc|}
\hline Iteration $k$ & 0 & 1 & 2 & 3 & $\cdots$ \\
\hline$J(I)$ & 0.50 & 0.07 & 0.023 & 0.018 & $\cdots$ \\
\hline \hline Iteration $k$ & $\cdots$ & 7 & 8 & & \\
\hline$J(I)$ & $\cdots$ & 0.0016 & 0.0007 & & \\
\hline
\end{tabular}

coordinates versus iteration $k$ are presented in Table 3. The error for the localization of the disk center is lower than $0.95 \mathrm{~mm}$. These previous results show that heat source coordinates are obtained after eight iterations with the measurement time equal to one second. The choice of this final time for identification purposes plays a crucial role in the convergence of the conjugate

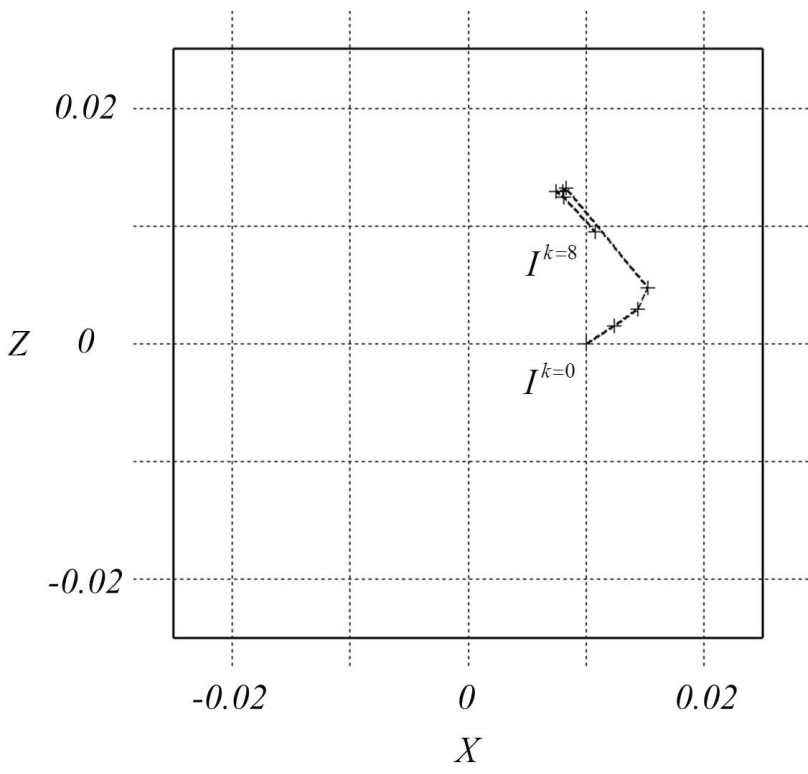

Fig. 6. Coordinate evolution on the heated face: Case A.1.

Table 3. Source coordinates (in mm) vs. iteration $k$ : Case A.1.

\begin{tabular}{|c||ccccc|}
\hline Iteration $k$ & 0 & 1 & 2 & 3 & $\cdots$ \\
\hline$x$ & 10 & 12.4 & 14.4 & 15.3 & $\cdots$ \\
\hline$z$ & 0 & 1.5 & 2.9 & 4.8 & $\cdots$ \\
\hline \hline Iteration $k$ & $\cdots$ & 7 & 8 & & \\
\hline$x$ & $\cdots$ & 8.1 & 10.8 & & \\
\hline$z$ & $\cdots$ & 12.5 & 9.5 & & \\
\hline
\end{tabular}

Table 4. Criterion and tracking error (in $\mathrm{mm}$ ) values versus iteration $k$ : Case A.2.

\begin{tabular}{|c||ccccc|}
\hline$k$ & 0 & 1 & 2 & 3 & 4 \\
\hline$J$ & 4.28 & 1.26 & 0.97 & 0.90 & 0.85 \\
\hline$T E$ & 10 & 9 & 8 & 5 & 7 \\
\hline \hline$k$ & 5 & 6 & 7 & $\cdots$ & 12 \\
\hline$J$ & 0.75 & 0.729 & 0.727 & $\cdots$ & 0.727 \\
\hline$T E$ & 6 & 7 & 6.8 & $\cdots$ & 0.5 \\
\hline
\end{tabular}

gradient algorithm. This choice depends on the number of information pieces provided by sensors. For noisy measurements, the final time has to be adapted in order to obtain the desired convergence (and the correct location).

4.2. Case A.2. The temperature predicted in the same configuration as for Case A.1 is disturbed by additive Gaussian noise defined by $\mathcal{N}(0,0.5)$.

Both the cost-function and tracking error versus iteration $k$ are shown in Table 4 and Fig. 7) It is shown that the convergence of the iterative algorithm is achieved in 12 iterations with the convergence of the tracking error under the threshold value $\mathrm{TE}\left(I^{k}, \theta\right)<10^{-3}$. In order to illustrate that the final time $t_{f}=1 \mathrm{~s}$ does not provide enough observations, tracking errors are compared for 


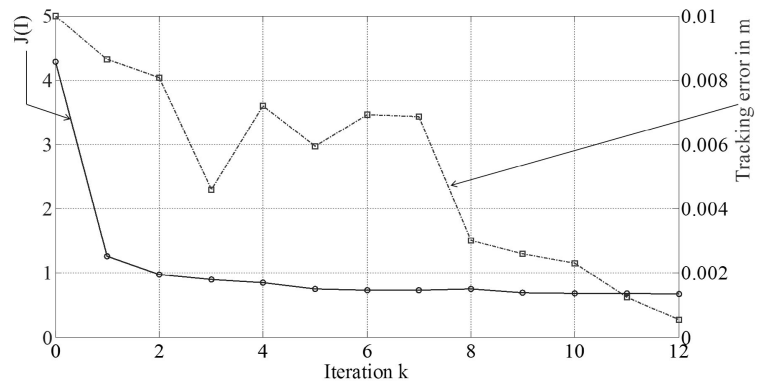

Fig. 7. Cost function and the tracking error: Case A.2.

Table 5. Source coordinates (in mm) vs. iteration $k$ : Case A.2.

\begin{tabular}{|c||ccccc|}
\hline$k$ & 0 & 1 & 2 & 3 & 4 \\
\hline$x$ & 10 & 12.8 & 14.6 & 14.2 & 6.7 \\
\hline$z$ & 0 & 1.8 & 3.3 & 8.2 & 16.4 \\
\hline \hline$k$ & 5 & $\cdots$ & 12 & & \\
\hline$x$ & 5.2 & $\cdots$ & 8.9 & & \\
\hline$z$ & 13.5 & $\cdots$ & 10.5 & & \\
\hline
\end{tabular}

$t_{f}=1 \mathrm{~s}$ and $t_{f}=2 \mathrm{~s}$ in Fig. 8 It is shown that for $t_{f}=1$ $\mathrm{s}$, the tracking error is almost constant for $k \geq 6$ and it is not possible to identify the correct heat source location. For $t_{f}=2 \mathrm{~s}$ the tracking error obtained after 12 iterations is lower than the desired accuracy. Coordinate evolution is shown in Table 5. The error for the localization of the disk center is lower than $1.21 \mathrm{~mm}$.

4.3. Case A.3. Let us consider a greater standard deviation, i.e., Gaussian noise $\mathcal{N}(0,1)$ (see Fig. 9). Both the cost-function and the tracking error versus iteration $k$ are shown in Table 6 and Fig. 10. The identified source coordinates are presented in Table 7 . The error for the localization of the disk center is lower than $0.77 \mathrm{~mm}$. Moreover, in Fig. 11, evolution of the tracking error for several final time values is presented. The obtained results show that three seconds $\left(t_{f}=3 \mathrm{~s}\right)$ are sufficient to identify the heating source coordinates considering the measured temperature disturbed by Gaussian noise $\mathcal{N}(0,1)$, while 2 seconds do not provide enough disturbed observations to

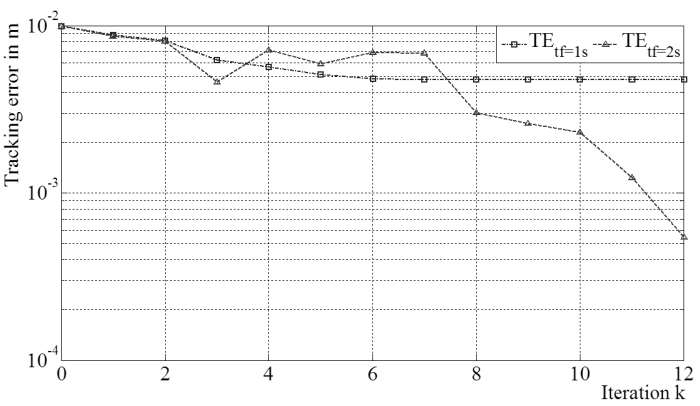

Fig. 8. Tracking error for different final time values: Case A.2.

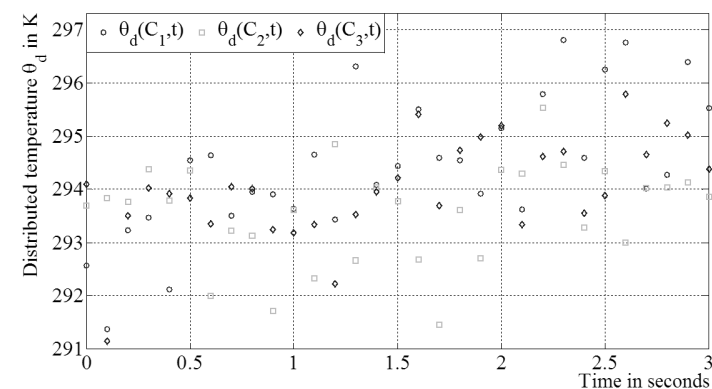

Fig. 9. Disturbed measurements for $t_{f}=3 \mathrm{~s}$ : Case A.3.

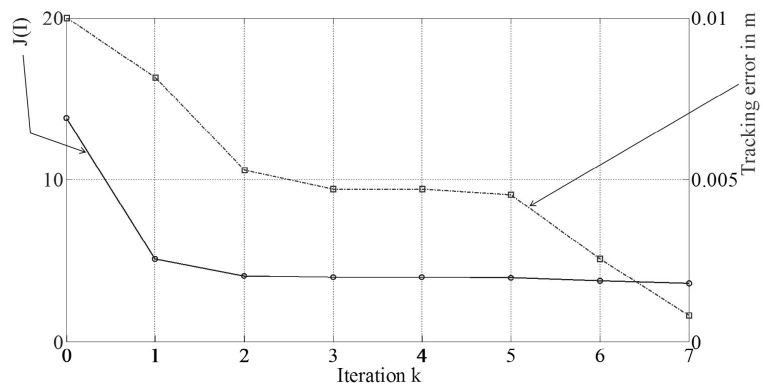

Fig. 10. Cost function and the tracking error: Case A.3.

identify the correct location.

The previous cases confirm robustness of the CGM when identifying a single heat source location. Moreover, the regularization effect of the CGM is illustrated when noisy data are taken into account for identification purposes.

Table 6. Criterion and tracking error (in $\mathrm{mm}$ ) values vs. iteration $k$ : Case A.3.

\begin{tabular}{|c||ccccc|}
\hline$k$ & 0 & 1 & 2 & 3 & 4 \\
\hline$J$ & 13.81 & 5.105 & 4.074 & 3.968 & 3.968 \\
\hline$T E$ & 10 & 8 & 5 & 4.7 & 4.7 \\
\hline \hline$k$ & 5 & 6 & 7 & & \\
\hline$J$ & 3.958 & 3.755 & 3.596 & & \\
\hline$T E$ & 4.5 & 2.6 & 0.8 & & \\
\hline
\end{tabular}

Table 7. Source coordinates vs. iteration $k$ : Case A.3.

\begin{tabular}{|c||ccccc|}
\hline$k$ & 0 & 1 & 2 & 3 & 4 \\
\hline$x$ & 10 & 12.9 & 13.7 & 12.4 & 12.4 \\
\hline$z$ & 0 & 2.4 & 6.2 & 5.9 & 5.9 \\
\hline \hline$k$ & 5 & 6 & 7 & & \\
\hline$x$ & 12 & 10.3 & 10.3 & & \\
\hline$z$ & 6 & 7.4 & 9.3 & & \\
\hline
\end{tabular}

4.4. Case B.1. Consider two heat sources whose real positions are defined by $I_{1}(0.01,-e / 2,0.01)$ and 


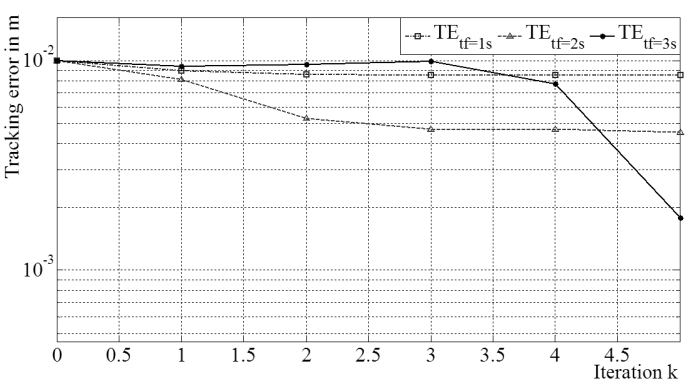

Fig. 11. Tracking error for different final time values: Case A.3.

Table 8. Criterion values vs. iteration $k$ : Case B.1

\begin{tabular}{|c||ccccc|}
\hline Iteration $k$ & 0 & 1 & 2 & 3 & 4 \\
\hline$J\left(I_{1}, I_{2}\right)$ & 1.14 & 0.18 & 0.074 & 0.058 & 0.047 \\
\hline \hline Iteration $k$ & $\cdots$ & 8 & 9 & & \\
\hline$J\left(I_{1}, I_{2}\right)$ & $\cdots$ & 0.0016 & $410^{-5}$ & & \\
\hline
\end{tabular}

$I_{2}(-0.01,-e / 2,0.01)$ in meters (see Fig. 12). Simulated temperatures (treated as measured temperatures for identification purposes) are presented in Fig. 13 .

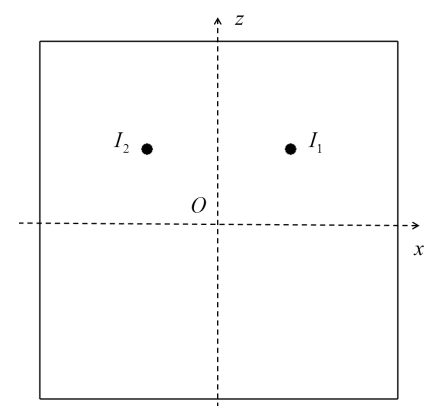

Fig. 12. Source location $y=-e / 2$ : Case B.1.

Assume that for the inverse problem solution the CGM is implemented with initial sources coordinates equal to $I_{1}^{k=0}(0.01,-e / 2,0)$ and $I_{2}^{k=0}(-0.01,-e / 2,0)$. Results are presented in Fig. 14 and Table 8. Evolution of the identified coordinates for the two heat sources is shown in Fig. 15 and the values are presented in Table 9. The error for the localization of the disk center is lower than $0.1 \mathrm{~mm}$. In this case, observations performed during only one second $\left(t_{f}=1 \mathrm{~s}\right)$ are relevant to identify the locations of two sources. The effect of noisy measurements is investigated in the following section.

4.5. Case B.2. Assume that the measured temperatures are disturbed by Gaussian noise $\mathcal{N}(0,0.5)$ (see Fig. 16). Evolution of both the cost function and the tracking error is outlined in Fig. 17 and Table 10. The obtained coordinates of the localizations of the two heating sources are given in Table 11. The error for the localization of the disk center is lower than $0.81 \mathrm{~mm}$. In order to justify the

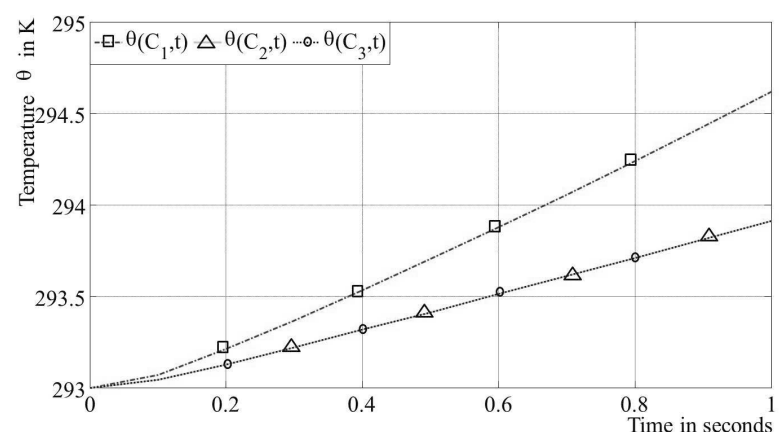

Fig. 13. Direct problem solution: Case B.1.

choice of the final time $\left(t_{f}=2 \mathrm{~s}\right)$, a comparison between the tracking errors obtained for $t_{f}=1 \mathrm{~s}$ and $t_{f}=2$ $\mathrm{s}$ versus iteration $k$ is presented in Fig. 17. It is shown that, for noise $\mathcal{N}(0,0.5)$, observations performed during 1 second are not efficient for identifying the locations of two heat sources. In fact, in such a situation the signal/noise ratio is too small and the CGM fails to extract relevant information so quickly. For a final time equal to 2 seconds, convergence is correct and unknown coordinates are accurately identified.

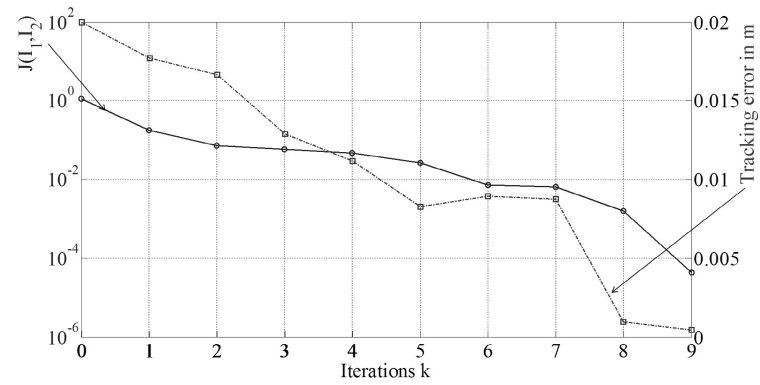

Fig. 14. Cost function and the tracking error: Case B.1.

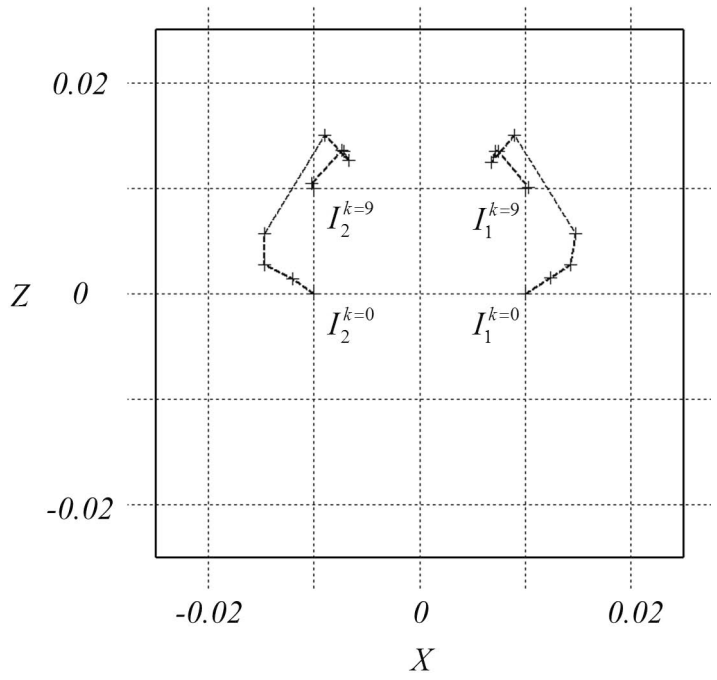

Fig. 15. Source coordinates evolution on the heated face: Case B.1. 
Table 9. Source coordinates (in mm) vs. iteration $k$ : Case B.1.

\begin{tabular}{|c||ccccc|}
\hline$k$ & 0 & 1 & 2 & 3 & 4 \\
\hline$x_{1}$ & 10 & 12.4 & 14.3 & 14.8 & 9 \\
$z_{1}$ & 0 & 1.5 & 2.8 & 5.7 & 15 \\
\hline$x_{2}$ & -10 & -12 & -14.7 & -14.7 & -9 \\
$z_{2}$ & 0 & 1.4 & 2.8 & 5.7 & 15 \\
\hline \hline$k$ & 5 & 6 & 7 & 8 & 9 \\
\hline$x_{1}$ & 6.8 & 7.2 & 7.5 & 10.3 & 10 \\
$z_{1}$ & 12.5 & 13.5 & 13.5 & 10.4 & 10 \\
\hline$x_{2}$ & -6.7 & -7.2 & -7.4 & -10.2 & -10 \\
$z_{2}$ & 12.6 & 13.5 & 13.6 & 10.5 & 10 \\
\hline
\end{tabular}

4.6. Case B.3. For a larger noise standard deviation $\mathcal{N}(0,1)$, the predicted temperature is shown in Fig. 19 . The CGM for of the identification source coordinates is called for $t_{f}=10 \mathrm{~s}$ and results are presented in Fig. 20 and Tables 12 and 13. The localization error of the disk center is lower than $1.21 \mathrm{~mm}$. According to the

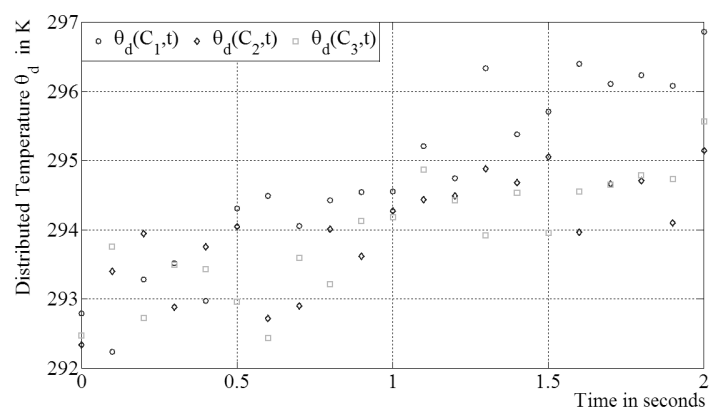

Fig. 16. Disturbed measurements for $t_{f}=2 \mathrm{~s}$ : Case B.2.

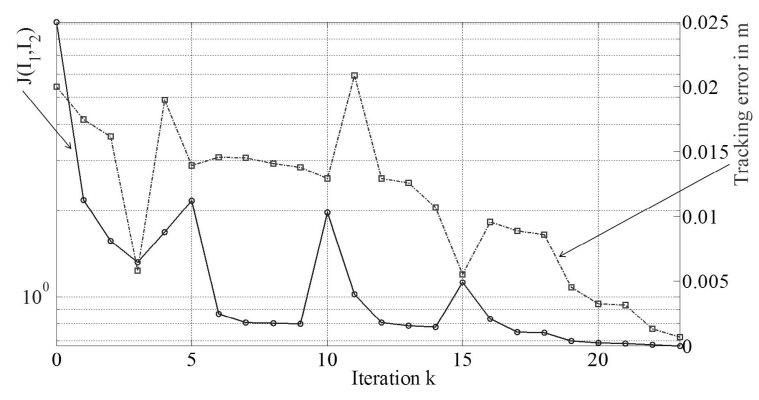

Fig. 17. Cost function and the tracking error vs. $k$ : Case B.2.

Table 10. Cost function and tracking error (in mm) vs. $k$ : Case B.2.

\begin{tabular}{|c||ccccc|}
\hline$k$ & 0 & 1 & 2 & 3 & 4 \\
\hline$J$ & 9.2 & 2.2 & 1.6 & 1.3 & 1.7 \\
\hline$T E$ & 20 & 17 & 16 & 6 & 20 \\
\hline \hline$k$ & 5 & 6 & $\cdots$ & 23 & \\
\hline$J$ & 2.17 & 0.087 & $\cdots$ & 0.67 & \\
\hline$T E$ & 14 & 14 & $\cdots$ & 0.6 & \\
\hline
\end{tabular}

Table 11. Source coordinates (in mm) vs. iteration $k$ : Case B.2.

\begin{tabular}{|c||ccccc|}
\hline$k$ & 0 & $\cdots$ & 3 & 4 & 5 \\
\hline$x_{1}$ & 10 & $\cdots$ & 12.7 & 7 & 4.1 \\
$z_{1}$ & 0 & $\cdots$ & 11.5 & 19 & 13.3 \\
\hline$x_{2}$ & -10 & $\cdots$ & -12.3 & -6 & -3.3 \\
$z_{2}$ & 0 & $\cdots$ & 11.5 & 19 & 12.6 \\
\hline \hline$k$ & $\cdots$ & 10 & 11 & 12 & $\cdots$ \\
\hline$x_{1}$ & $\cdots$ & 15.3 & 3.5 & 6 & $\cdots$ \\
$z_{1}$ & $\cdots$ & 8.4 & 17.6 & 15 & $\cdots$ \\
\hline$x_{2}$ & $\cdots$ & -17.3 & -1.6 & -5 & $\cdots$ \\
$z_{2}$ & $\cdots$ & 9.4 & 16.9 & 15 & $\cdots$ \\
\hline \hline$k$ & 15 & 16 & 17 & $\cdots$ & 23 \\
\hline$x_{1}$ & 12 & 8 & 7.5 & $\cdots$ & 9.6 \\
$z_{1}$ & 9 & 14.3 & 13.5 & $\cdots$ & 10.7 \\
\hline$x_{2}$ & -14 & -7.3 & -6.7 & $\cdots$ & -9.6 \\
$z_{2}$ & 10 & 13.9 & 13.1 & $\cdots$ & 10.3 \\
\hline
\end{tabular}

previous results with the final time $t_{f}=10 \mathrm{~s}$, it is shown that the CGM is robust and efficient when identifying the

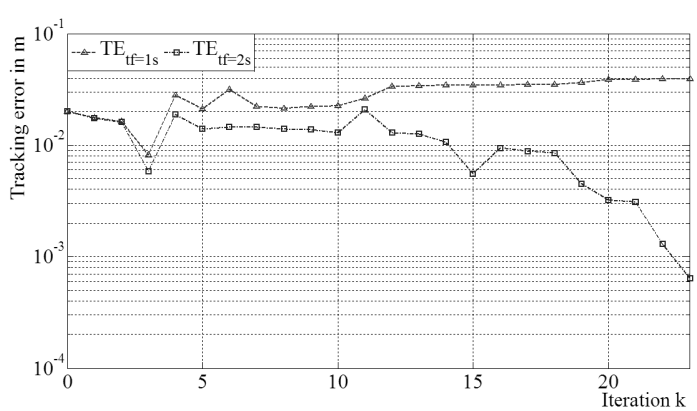

Fig. 18. Tracking error for different final times: Case B.2.

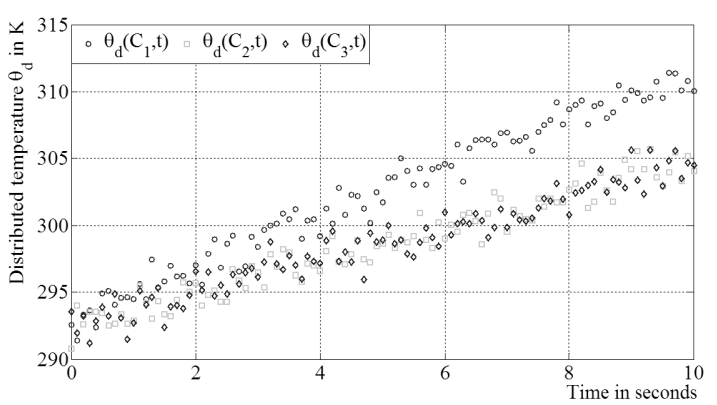

Fig. 19. Disturbed measurements for $t_{f}=10 \mathrm{~s}$ : Case B.3.

Table 12. Cost function and the tracking error (in MM) vs. $k$ : Case B.3.

\begin{tabular}{|c||ccccc|}
\hline$k$ & 0 & $\cdots$ & 3 & 4 & 5 \\
\hline$J$ & 384 & $\cdots$ & 50 & 40 & 27 \\
\hline$T E$ & 20 & $\cdots$ & 7 & 14 & 12 \\
\hline \hline$k$ & $\cdots$ & 7 & 8 & $\cdots$ & 11 \\
\hline$J$ & $\cdots$ & 26.37 & 26.37 & $\cdots$ & 12.84 \\
\hline$T E$ & $\cdots$ & 8.5 & 3.5 & $\cdots$ & 0.5 \\
\hline
\end{tabular}


locations of two heat sources. In order to justify the choice $t_{f}=10 \mathrm{~s}$, several final times are compared in Fig. 21 It is important to note that for observations performed during 10 seconds, even with significant noise (see Fig. 19, heat source coordinates are accurately identified in only 11 iterations.

4.7. Case C.1. For this last studied configuration, the CGM is implemented in order to identify the location of three heat sources $\left(S_{1}, S_{2}\right.$ and $\left.S_{3}\right)$ located at $I_{1}(0.01,-e / 2,0.01), I_{2}(-0.01,-e / 2,0.01)$ and $I_{3}(0,-e / 2,-0.005)$ in meters (see Fig. 22).

Simulated temperatures (treated as the measured temperatures for identification purposes) are presented in Fig. 23. The corresponding initial source coordinates are $I_{1}^{k=0}(0.01,-e / 2,0), \quad I_{2}^{k=0}(-0.01,-e / 2,0) \quad$ and $I_{3}^{k=0}(0,-e / 2,0)$.

The results are presented in Figs. 24 and 25 and Tables 14 and 15. The error for the localization of the disk center is lower than $0.23 \mathrm{~mm}$. For this situation, if the final time is lower than $7 \mathrm{~s}$, then the algorithm cannot converge and the cost function cannot decrease under the stopping criterion fixed as $J_{\text {stop }}=10^{-3}$ (see Section 3.5). In fact, an important amount of observations is required in order to discriminate between the three sources.

4.8. Case C.2. Measurements disturbed by Gaussian noise $\mathcal{N}(0,0.5)$ are considered in Fig. 26, The results are shown in Fig. 27 and Tables 16 and 17. The error for the localization of the disk center is lower than $0.65 \mathrm{~mm}$.

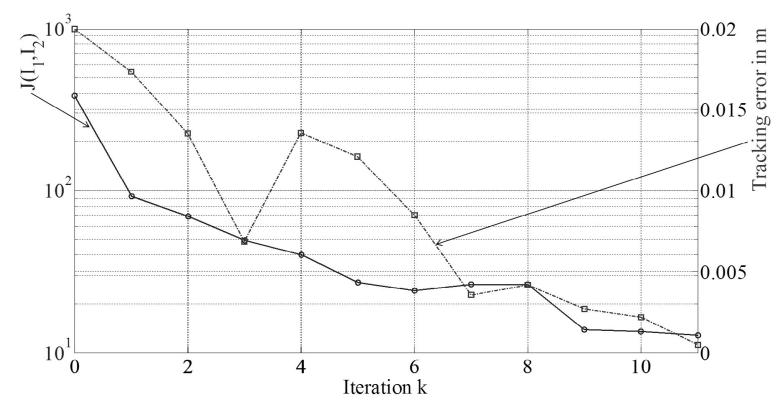

Fig. 20. Cost function and tracking error evolution: Case B.3.

Table 13. Source coordinates (in $\mathrm{mm}$ ) vs. iteration $k$ : Case B.3.

\begin{tabular}{|c||ccccc|}
\hline$k$ & 0 & 1 & 2 & 3 & 4 \\
\hline$x_{1}$ & 10 & 13.8 & 15 & 11 & 6.4 \\
$z_{1}$ & 0 & 2.2 & 5 & 13 & 15.7 \\
\hline$x_{2}$ & -10 & -13.8 & -15 & -10 & -6.1 \\
$z_{2}$ & 0 & 2.2 & 5 & 13 & 15.6 \\
\hline \hline$k$ & 5 & 6 & $\cdots$ & 11 & \\
\hline$x_{1}$ & 5.8 & 7.9 & $\cdots$ & 9.5 & \\
$z_{1}$ & 14.3 & 13.8 & $\cdots$ & 11.1 & \\
\hline$x_{2}$ & -5.66 & -7.8 & $\cdots$ & -9.5 & \\
$z_{2}$ & 14.2 & 13.6 & $\cdots$ & 10.8 & \\
\hline
\end{tabular}

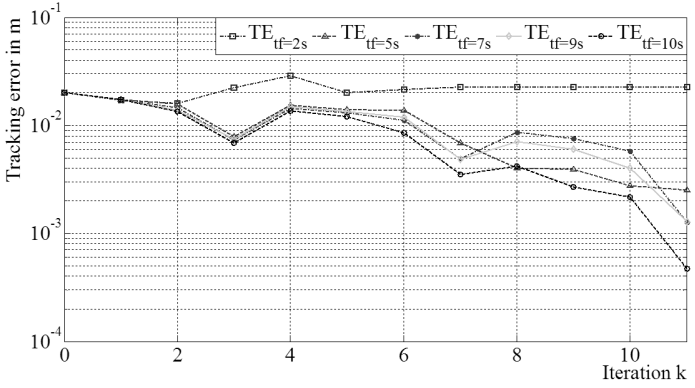

Fig. 21. Tracking error for different final times: Case B.3.

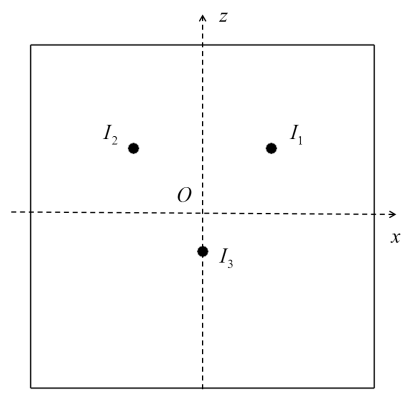

Fig. 22. Source location $y=-e / 2$ : Case C.1.

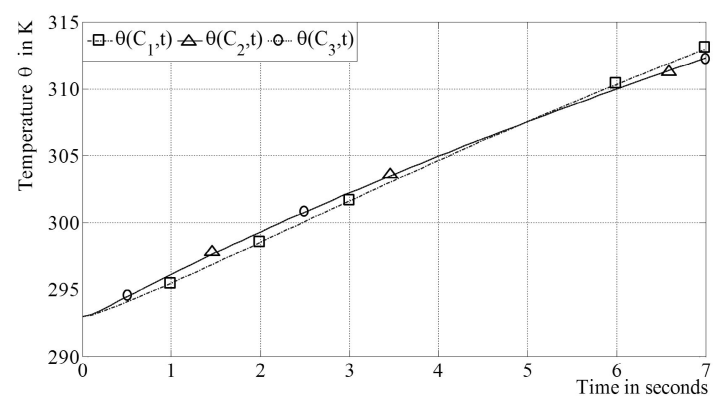

Fig. 23. Direct problem solution: Case C.1.

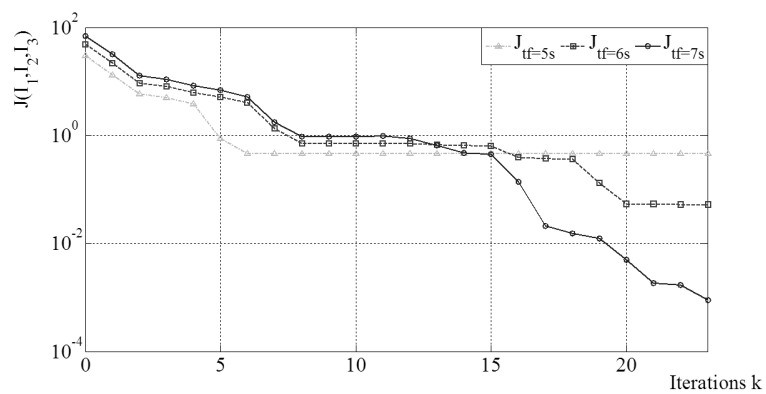

Fig. 24. Cost function and the tracking error vs. $k$ : Case C.1.

Table 14. Criterion values vs. iteration $k$ : Case C.1.

\begin{tabular}{|c||cccccc|}
\hline$k$ & 0 & 1 & 2 & $\cdots$ & 22 & 23 \\
\hline$J$ & 70 & 32 & 13 & $\cdots$ & 0.002 & 0.0009 \\
\hline
\end{tabular}

In Fig. 28, evolution of the tracking error is shown for several final times, and it is evidenced that a time longer than for non-disturbed observations is required for 


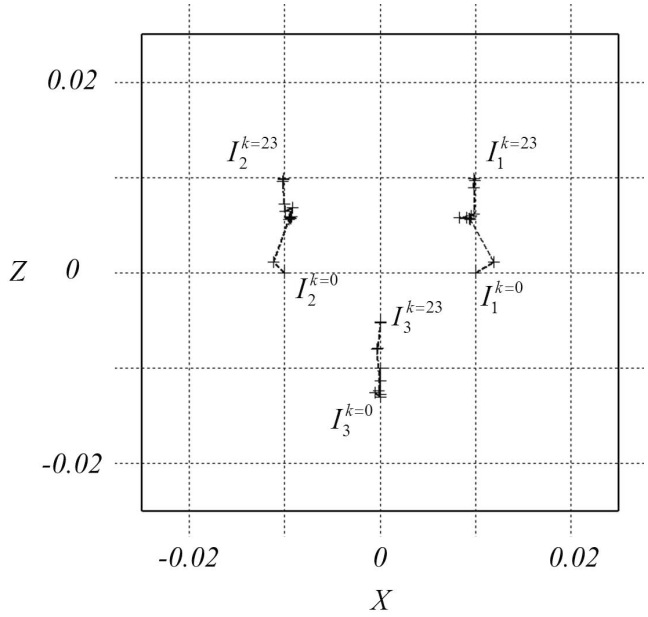

Fig. 25. Evolution of the source coordinates on the heated face: Case C.1.

Table 15. Source coordinates (in mm) vs. iteration $k$ : Case C.1.

\begin{tabular}{|c||ccccccc|}
\hline$k$ & 0 & 1 & 2 & $\cdots$ & 17 & $\cdots$ & 23 \\
\hline$x_{1}$ & 10 & 11.9 & 9.5 & $\cdots$ & 9.9 & $\cdots$ & 9.8 \\
$z_{1}$ & 0 & 1.1 & 5.6 & $\cdots$ & 9.7 & $\cdots$ & 9.9 \\
\hline$x_{2}$ & -10 & -11.2 & -9.6 & $\cdots$ & -10.2 & $\cdots$ & -10.1 \\
$z_{2}$ & 0 & 1.1 & 5.6 & $\cdots$ & 9.6 & $\cdots$ & 9.9 \\
\hline$x_{3}$ & 0 & 0 & -0.01 & $\cdots$ & 0 & $\cdots$ & 0 \\
$z_{3}$ & -10 & -11.3 & -12.4 & $\cdots$ & -5.1 & $\cdots$ & -5.2 \\
\hline
\end{tabular}

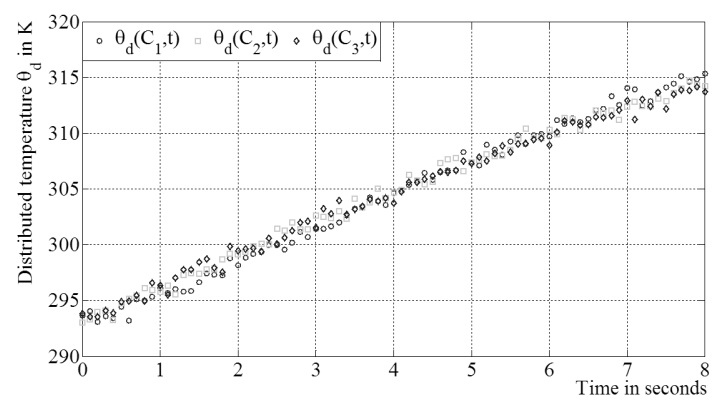

Fig. 26. Disturbed measurements for $t_{f}=8 \mathrm{~s}$ : Case C.2.

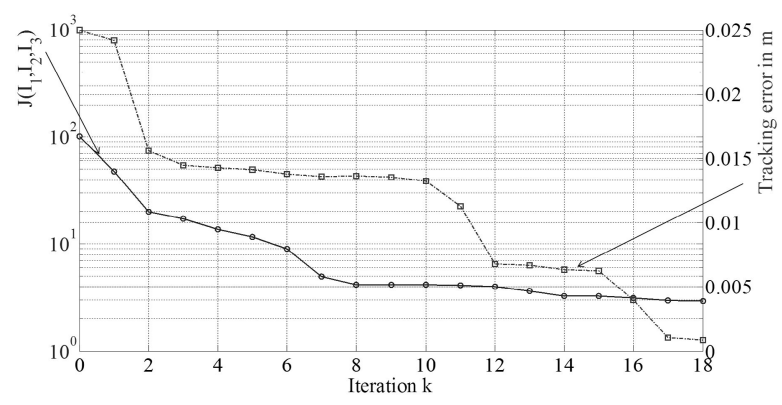

Fig. 27. Cost function and the tracking error: Case C.2.

location identification.

4.9. Case C.3. For this final case, additive Gaussian noise $\mathcal{N}(0,1)$ is considered, and three sensors and three heat sources have to be located. The measurements are
Table 16. Cost function and the tracking error (in $\mathrm{mm}$ ) vs. iteration $k$ : Case C.2.

\begin{tabular}{|c||ccccccc|}
\hline$k$ & 0 & 1 & 2 & 3 & 4 & $\cdots$ & 18 \\
\hline$J$ & 101 & 47 & 20 & 17 & 14 & $\cdots$ & 2.96 \\
\hline$T E$ & 25 & 24 & 16 & 14.4 & 14.2 & $\cdots$ & 0.8 \\
\hline
\end{tabular}

Table 17. Source coordinates (in $\mathrm{mm}$ ) versus iteration $k$ : Case

\begin{tabular}{l||cccccc|} 
C.2. \\
\begin{tabular}{|c||cccccc|}
\hline$k$ & 0 & 1 & 2 & $\cdots$ & 11 & 12 \\
\hline$x_{1}$ & 10 & 11.3 & 9.5 & $\cdots$ & 9.6 & 10 \\
$z_{1}$ & 0 & 1.2 & 6.0 & $\cdots$ & 6.3 & 7.6 \\
\hline$x_{2}$ & -10 & -11.1 & -9.3 & $\cdots$ & -9.2 & -9.6 \\
$z_{2}$ & 0 & 1.2 & 5.9 & $\ldots$ & 6.2 & 7.4 \\
\hline$x_{3}$ & 0 & 0 & 0 & $\cdots$ & 0 & -0.07 \\
$z_{3}$ & -10 & -10 & -11.4 & $\ldots$ & -8.8 & -6.8 \\
\hline \hline$k$ & $\cdots$ & 15 & $\ldots$ & 17 & 18 & \\
\hline$x_{1}$ & $\cdots$ & 9.6 & $\cdots$ & 9.9 & 9.9 & \\
$z_{1}$ & $\ldots$ & 8.1 & $\ldots$ & 8.9 & 10.2 & \\
\hline$x_{2}$ & $\cdots$ & -9.9 & $\cdots$ & -10.9 & -10.5 & \\
$z_{2}$ & $\ldots$ & 7.9 & $\ldots$ & 9.4 & 9.6 & \\
\hline$x_{3}$ & $\cdots$ & 0.09 & $\cdots$ & -0.01 & 0.02 & \\
$z_{3}$ & $\ldots$ & -7.2 & $\ldots$ & -5.3 & -5.1 & \\
\hline
\end{tabular}
\end{tabular}

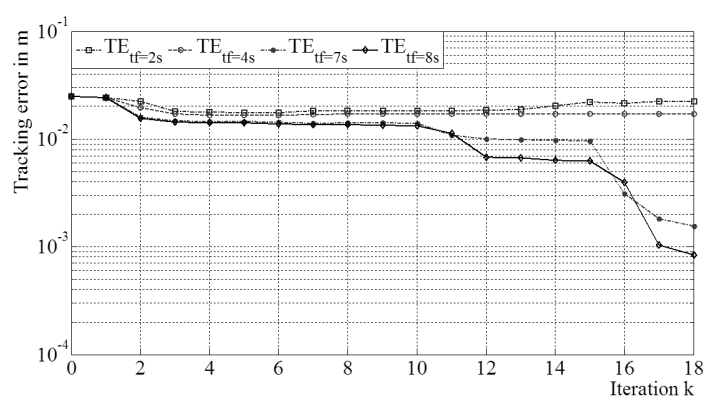

Fig. 28. Tracking error evolution for different final time values: Case C.2.

shown in Fig. 29. The results are shown in Fig. 30 as well as Tables 18 and 19 for the final time $t_{f}=16 \mathrm{~s}$. The error for the localization of the disk center is lower than 0.45 $\mathrm{mm}$. In Fig. 31, evolution of the tracking error is shown for several final times.

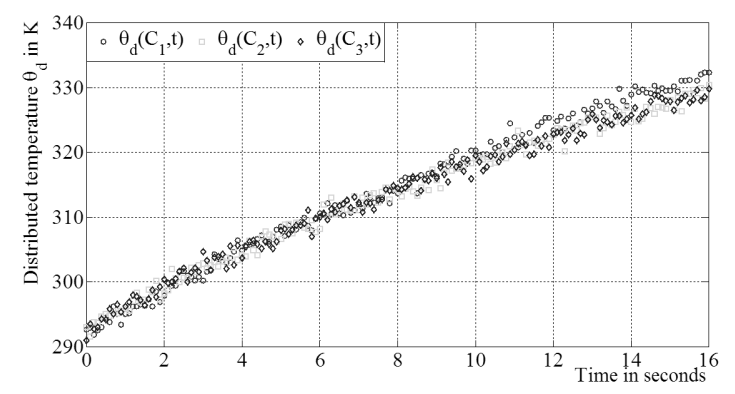

Fig. 29. Disturbed measurements for $t_{f}=16 \mathrm{~s}$ : Case C.3. 
If $t_{f}<16 \mathrm{~s}$, the tracking error does not converge under the desired threshold.

\section{Analysis}

According to the previous results, the necessary measurement time $t_{f}$ depends on the standard deviation of the noise and $2 N_{s}$ unknown parameters. This can be illustrated by Table 20. In realistic cases, where the tracking error TE cannot be estimated, it is more difficult to define the reduced time $t_{f}$. It seems to be attractive to investigate the signal/noise ratio and to compare the energy signal

$$
\mathcal{E}=\int_{0}^{t_{f}} \sum_{i=1}^{3}\left(\hat{\theta}_{i}(t)-\theta_{0}\right)^{2} \mathrm{~d} t
$$

with the admissible level of minimization

$$
J_{\text {stop }}=\frac{3}{2} \sigma^{2} n_{t} .
$$

It is important to notice that both of these functions are increasing in time and are obtained without any a priori information on the source location. Without noise, it is important to obtain enough information in order to discriminate between sources. The final time depends on the distance between sources and sensors, as well as the heat flux. In the three studied configurations, this time can be described by $t_{f}=1+t_{0}$, where $t_{0}$ is defined as the time for which $\mathcal{E}>e^{7.5\left(N_{s}-2\right)}$. If noise has to be taken into account, then $t_{f}=1+t_{0}+t_{1}$, where $t_{1}$ is defined as the time for which $\mathcal{E}>\left(J_{\text {stop }}\right)^{0.6 N_{s}}$.

It is obvious that the previous formulas are heuristically determined and adapted to the studied configurations. However, the complexity problem is taken into account: $t_{0}$ depends on the number of sources and $t_{1}$ is related to the number of observations and the standard

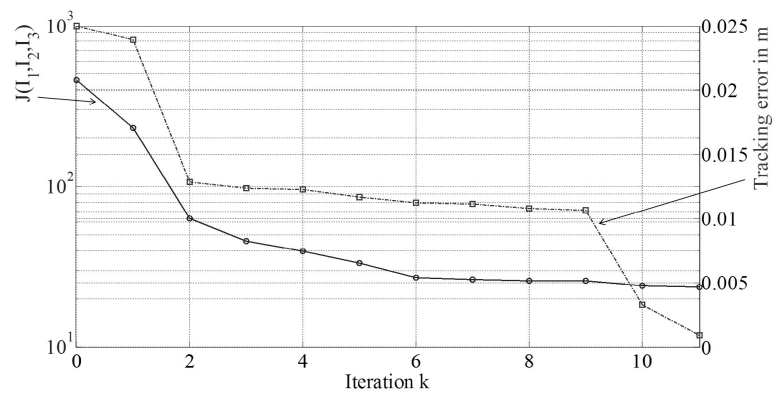

Fig. 30. Cost function and the tracking error: Case C.3.

Table 18. Cost function and the tracking error (in $\mathrm{mm}$ ) vs. iteration $k$ : Case C.3.

\begin{tabular}{|c||ccccccc|}
\hline$k$ & 0 & 1 & 2 & 3 & 4 & $\cdots$ & 11 \\
\hline$J$ & 457 & 231 & 63 & 46 & 40 & $\cdots$ & 23.6 \\
\hline$T E$ & 25 & 24 & 13 & 12.3 & 12.2 & $\cdots$ & 0.9 \\
\hline
\end{tabular}

Table 19. Source coordinates (in mm) vs. iteration $k$ : Case C.3.

\begin{tabular}{|c||ccccccc|}
\hline$k$ & 0 & 1 & 2 & $\cdots$ & 9 & 10 & 11 \\
\hline$x_{1}$ & 10 & 11.5 & 8.9 & $\cdots$ & 9.0 & 9.4 & 9.6 \\
$z_{1}$ & 0 & 1.4 & 7.2 & $\cdots$ & 7.0 & 9.1 & 9.9 \\
\hline$x_{2}$ & -10 & -11.5 & -9.0 & $\cdots$ & -9.2 & -9.1 & -10 \\
$z_{2}$ & 0 & 1.5 & 7.1 & $\cdots$ & 7 & 9.1 & 9.9 \\
\hline$x_{3}$ & 0 & 0 & 0.01 & $\cdots$ & 0.02 & 0.4 & -0.4 \\
$z_{3}$ & -10 & -11.5 & -11.8 & $\cdots$ & -9.4 & -6.3 & -5.2 \\
\hline
\end{tabular}

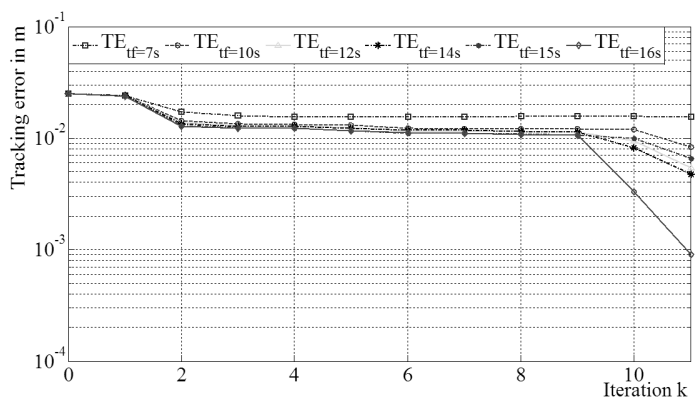

Fig. 31. Tracking error for different final times $t_{f}$ : Case C.3.

Table 20. Required measurement time $t_{f}$ (in s).

\begin{tabular}{|c||ccc|}
\hline & $\sigma=0$ & $\sigma=0.5$ & $\sigma=1$ \\
\hline$N_{s}=1$ & 1 & 2 & 3 \\
\hline$N_{s}=2$ & 1 & 2 & 10 \\
\hline$N_{s}=3$ & 7 & 8 & 16 \\
\hline
\end{tabular}

Table 21. Admissible level of minimization and signal energy (in $\mathrm{K}^{2} \cdot \mathrm{s}$ ).

\begin{tabular}{|c||ccc|}
\hline$J_{\text {stop }}$ & $\sigma=0$ & $\sigma=0.5$ & $\sigma=1$ \\
\hline$N_{s}=1$ & 0 & 7.5 & 45 \\
\hline$N_{s}=2$ & 0 & 7.5 & 150 \\
\hline$N_{s}=3$ & 0 & 30 & 240 \\
\hline \hline $\mathcal{E}$ & $\sigma=0$ & $\sigma=0.5$ & $\sigma=1$ \\
\hline$N_{s}=1$ & 0.4 & 3 & 10 \\
\hline$N_{s}=2$ & 1.4 & 11 & 1400 \\
\hline$N_{s}=3$ & 2800 & 4200 & 33400 \\
\hline
\end{tabular}

Table 22. Predicted $t_{f}$ (in s).

\begin{tabular}{|c||ccc|}
\hline \multicolumn{1}{|c||}{} & $\sigma=0$ & $\sigma=0.5$ & $\sigma=1$ \\
\hline$N_{s}=1$ & 1 & 3 & 4 \\
\hline$N_{s}=2$ & 2 & 4 & 7 \\
\hline$N_{s}=3$ & 7 & 8 & 17 \\
\hline
\end{tabular}

deviation of the noise. In Table 22, predicted values $t_{f}=1+t_{0}+t_{1}$ are given and can be compared with Table 20.

\section{Conclusion}

In this paper, an inverse heat conduction problem (IHCP) is formulated in order to estimate the location of one or several heat sources in a three-dimensional 
geometry using the conjugate gradient method (CGM). For this algorithm implementation, the solution of three well-posed problems is required. Numerical experiments have been performed in order to study the effect of the number of sources, measurement noise, regularization, and the observation time required for accurate identification. Robustness of the proposed algorithm has been illustrated. If an adequate number of observations is available, identification is achieved in several iterations. This adequate number of observations is directly connected to the final time. In experimental situations, it has to be determined considering the signal/noise ratio, the number of sources and the admissible level of minimization.

Several directions can be considered for further works. Theoretical determination of the required observation time has to be investigated in specific academic situations. Simultaneous identification of both the location and the strength of fixed heat sources is also an interesting goal considering the necessary measurement time. Moreover, for mobile heat source tracking, a sliding time window based on minimal time determination seems to be quite an attractive approach. Last but not least, an experimental device will be developed in the LARIS-Lab research institute in order to test numerous situations for several heat sources.

\section{References}

Alifanov, O.M. (1994). Inverse Heat Transfer Problems, Springer-Verlag, Berlin.

Alifanov, O.M., Artyukhin, E.A. and Rumyantsev, S.V. (1995). Extreme Methods for Solving Ill Posed Problems with Applications to Inverse Heat Transfer Problems, Begell House, New York, NY.

Autrique, L., Chaussavoine, C., Leyris, J. and Ferriere, A. (2000). Optimal sensor strategy for parametric identification of a thermal system, Proceedings of IFACSYSID 2000, Santa Barbara, CA, USA.

Autrique, L., Leyris, J. and Ramdani, N. (2002). Optimal sensor location: An experimental process for the identification of moving heat sources, Proceedings of the 15th World Congress IFAC, Barcelona, Spain.

Beck, J. and Arnold, K. (1977). Parameter Estimation in Engineering and Science, John Wiley and Sons, New York, NY.

Chen, M., Berkowitz-Mattuck, J.B. and Glaser, P.E. (1963). The use of a kaleidoscope to obtain uniform flux over a large area in a solar or arc imaging furnace, Applied Optics 2(3): 265-271.

Daouas, N. and Radhouani, M. (2004). A new approach of the Kalman filter using future temperature measurements for nonlinear inverse heat conduction problem, Numerical Heat Transfer 45(6): 565-585.

Daouas, N. and Radhouani, M. (2007). Experimental validation of an extended Kalman smoothing technique for solving nonlinear inverse heat conduction problems, Inverse Problems in Science and Engineering 15(7): 765-782.

Egger, H., Heng, Y., Marquardt, W. and Mhamdi, A. (2009). Efficient solution of a three-dimensional inverse heat conduction problem in pool boiling, Inverse Problems 25(9), Article ID: 095006.

Girault, M., Videcoq, E. and Petit, D. (2010). Estimation of time-varying heat sources through inversion of a low order model built with the modal identification method from in-situ temperature measurements, Journal of Heat and Mass Transfer 53: 206-219.

Hager, W. and Zhang, H. (2006). A survey of nonlinear conjugate gradient method, Pacific Journal of Optimization 2(1): 35-58.

Hasanov, A. and Pektas, B. (2013). Identification of an unknown time-dependent heat source term from overspecified Dirichlet boundary data by conjugate gradient method, Computers and Mathematics with Applications $\mathbf{6 5}(1)$ : $42-57$.

Huang, C. and Chen, W. (1999). A three-dimensional inverse forced convection problem in estimating surface heat flux by conjugate gradient method, International Journal of Heat and Mass Transfer 43(17): 3171-3181.

Isakov, V. (1998). Inverse Problems for Partial Differential Equations, Springer-Verlag, New York, NY.

Jarny, Y., Ozisik, M. and Bardon, J. (1991). A general optimization method using adjoint equation for solving multidimensional inverse heat conduction, International Journal of Heat and Mass Transfer 34(11): 2911-2919.

Khachfe, R. and Jarny, Y. (2000). Numerical solution of 2-D nonlinear inverse heat conduction problems using finite-element techniques, Numerical Heat Transfer B 37(1): 45-67.

Khachfe, R. and Jarny, Y. (2001). Determination of heat sources and heat transfer coefficient for two-dimensional heat flow numerical and experimental study, International Journal of Heat and Mass Transfer 44(7): 1309-1322.

Kolodziej, J., Mierzwiczak, M. and Ciakowski, M. (2010). Application of the method of fundamental solutions and radial basis functions for inverse heat source problem in case of steady-state, International Communications in Heat and Mass Transfer 37(2): 121-124.

Lefèvre, F. and Le Niliot, C.L. (2002). Multiple transient point heat sources identification in heat diffusion: Application to experimental 2D problems, Journal of Heat and Mass Transfer 45(9): 1951-1964.

Le Niliot, C. and Lefèvre, F. (2001). A method for multiple steady line heat sources identification in diffusive system: Application to an experimental 2D problem, Journal of Heat and Mass Transfer 44(7): 1425-1438.

Le Niliot, C. and Lefèvre, F. (2004). A parameter estimation approach to solve the inverse problem of point heat sources identification, International Journal of Heat and Mass Transfer 47(4): 827-841. 
Lormel, C., Autrique, L. and Claudet, B. (2004). Mathematical modeling of skin behavior during a laser radiation exposure, Proceedings of the 2nd European Survivability Workshop, Noordwijk, The Netherlands.

Mechhoud, S., Witrant, E., Dugard, L. and Moreau, D. (2013). Combined distributed parameters and source estimation in tokamak plasma heat transport, Proceedings of the European Control Conference, Zurich, Switzerland.

Mierzwiczak, M. and Kolodziej, J. (2010). Application of the method of fundamental solutions and radial basis functions for inverse transient heat source problem, Computer Physics Communications 181(12): 2035-2043.

Mierzwiczak, M. and Kolodziej, J. (2011). The determination of heat sources in two dimensional inverse steady heat problems by means of the method of fundamental solutions, Inverse Problems in Science and Engineering 19: $777-792$.

Mierzwiczak, M. and Kolodziej, J. (2012). Application of the method of fundamental solutions with the Laplace transformation for the inverse transient heat source problem, Journal of Theoretical and Applied Mechanics 50(4): 1011-1023.

Mohammadiun, M., Rahimi, A. and Khazaee, I. (2011). Estimation of the time-dependent heat flux using the temperature distribution at a point by conjugate gradient method, International Journal of Thermal Sciences 50(12): 2443-2450.

Morozov, V. (1994). Methods for Solving Incorrectly Posed Problems, Springer-Verlag, New York, NY.

Museux, N., Perez, L., Autrique, L. and Agay, D. (2012). Skin burns after laser exposure: Histological analysis and predictive simulation, Burns 38(5): 658-667.

Park, H., Chung, O. and Lee, J. (1999). On the solution of inverse heat transfer problem using the Karhunen-Love-Galerkin method, International Journal of Heat and Mass Transfer 42: 127-142.

Perez, L., Gillet, M. and Autrique, L. (2007). Parametric identification of a multi-layered intumescent system, Proceedings of the 5th International Conference: Inverse Problems (Identification, Design and Control), Moscow, Russia.

Perez, L. and Vergnaud, A. (2016). Observation strategies for mobile heating source tracking, High Temperatures, High Pressures 45(1): 57-76.

Powell, M. (1977). Restart procedures for the conjugate gradient method, Mathematical Programming 12(1): 241-254.
Prudhomme, M. and Nguyen, T.H. (1998). On the iterative regularization of inverse heat conduction problems by conjugate gradient method, International Communications in Heat and Mass Transfer 25(7): 999-1008.

Rakotoniaina, J., Breitenstein, O. and Langenkamp, M. (2002). Localization of weak heat sources in electronic devices using highly sensitive lock-in thermography, Materials Science and Engineering B: Solid-State Materials for Advanced Technology 91: 481-485.

Renault, N., André, S., Maillet, D. and Cunat, D. (2008). A two-step regularized inverse solution for 2-D heat source reconstruction, Journal of Thermal Sciences 47(7): 827-841.

Renault, N., André, S., Maillet, D. and Cunat, D. (2010). A spectral method for the estimation of a thermomechanical heat source from infrared temperature measurements, Journal of Thermal Sciences 49(8): 1394-1406.

Rouquette, S., Autrique, L., Chaussavoine, C. and Thomas, L. (2007a). Identification of influence factors in a thermal model of a plasma-assisted chemical vapor deposition process, Inverse Problems in Science and Engineering 15(5): 489-515.

Rouquette, S., Guo, J. and Masson, P.L. (2007b). Estimation of the parameters of a Gaussian heat source by the Levenberg-Marquardt method: Application to the electron beam welding, International Journal of Thermal Sciences 46(2): 128-138.

Silva Neto, A. and Ozisik, M. (1993). Simultaneous estimation of location and timewise-varying strength of a plane heat source, Numerical Heat Transfer A 24(4): 467-477.

Tarantola, A. (2005). Inverse Problem Theory and Methods for Model Parameter Estimation, SIAM Society for Industrial and Applied Mathematics, Philadelphia, PA.

Weinstock, R.P. (1952). Calculus of Variations, McGraw, New York, NY.

Yi, Z. and Murio, D. (2002). Source term identification in 1D IHCP, Journal of Computers and Mathematics with Applications 47(10/11): 1921-1933.

Zhou, J., Zhang, Y., Chen, J. and Feng, Z. (2010) Inverse estimation of surface heating condition in a three-dimensional object using conjugate gradient method, International Journal of Heat and Mass Transfer 53: 2643-2654.

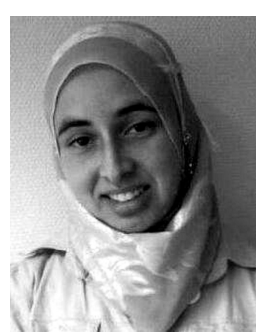

Sara Beddiaf received her Ph.D. degree in engineering sciences from the University of Angers in 2013. From October 2013 to March 2014, she held a temporary position at the University of Artois. Since April 2014, she has been a postdoctoral research fellow at the CNRS-Pprime Institute in Poitiers, France. 


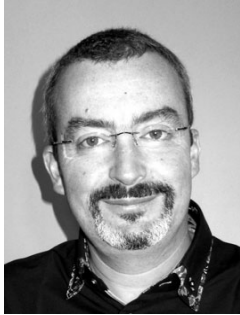

Laurent Autrique received a postgraduate degree (DEA) in process control from École Centrale (Nantes, France) in 1992, and a Ph.D. degree in the same institute in 1995 . He was a research scientist for the PROMES Research Institute (CNRS Perpignan, France) until 2002. Then, from 2002 to 2007, he was at the EHF Department (Expertise Hauts Flux), DGA (Weaponry Department of the French Ministry of Defence), Font Romeu (France). He is currently a professor in LARIS (ISTIA, University of Angers, France). His research works are devoted to parametric identification, inverse problems, and process analysis. His main publications are focused on systems of nonlinear PDEs describing state evolution of complex thermal processes.

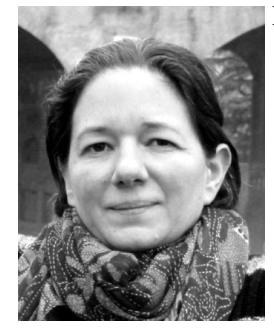

Laetitia Perez received her postgraduate degree in process engineering (University of Perpignan, France) in 2000 and her Ph.D. degree from Ecole Nationale Supérieure des Arts et Métiers (ENSAM, France) in 2003. From 2004 to 2006 she held a temporary research monitoring position for the French Ministry of Defence in FontRomeu. In 2006, she joined the Thermokinetic Laboratory of Nantes, where she is currently an associate professor. Since 2012, she has been the head of a department of her teaching institute. Her research interests are in the modelling of thermal processes, development of experimental test beds and solution of inverse problems.

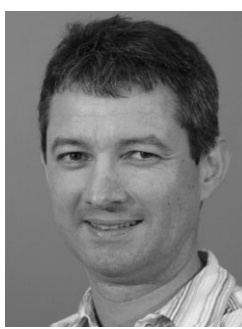

Jean-Claude Jolly received a postgraduate degree (DEA) in mechanical physics from the University of Paris 6 in 1992. He succeeded to the aggregation of mathematics in 1993 and joined the University of Angers in 1994 as an associate professor at ISTIA, a school of engineering. $\mathrm{He}$ received a $\mathrm{Ph} . \mathrm{D}$. degree in pure mathematics from this university in 2001 and became an assistant professor in 2003. He is currently a researcher in LARIS. After some work on hybrid systems, he has focused his activities on inverse problems and control theory for distributed parameter systems.

\section{Appendix}

In order to better understand the formulation of the boundary and final conditions in the adjoint problem, consider the variation in the Lagrangian

$$
\begin{aligned}
\delta \mathcal{L}(\theta, I, \psi)= & \int_{0}^{t_{f}} \int_{\Omega} E \delta \theta \mathrm{d} t \mathrm{~d} \Omega \\
& +\int_{0}^{t_{f}} \int_{\Omega}\left(\rho c \frac{\partial \delta \theta}{\partial t}-\lambda \Delta \delta \theta\right) \psi \mathrm{d} t \mathrm{~d} \Omega \\
= & \mathcal{I}_{1}+\mathcal{I}_{2} .
\end{aligned}
$$

The second term is developed using integration by parts in time and space:

$$
\mathcal{I}_{2}=\int_{0}^{t_{f}} \int_{\Omega}\left(\rho c \frac{\partial \delta \theta}{\partial t}-\lambda \Delta \delta \theta\right) \psi \mathrm{d} t \mathrm{~d} \Omega
$$

$$
\begin{aligned}
& =\int_{0}^{t_{f}} \int_{\Omega} \rho c \frac{\partial \delta \theta}{\partial t} \psi \mathrm{d} t \mathrm{~d} \Omega-\int_{0}^{t_{f}} \int_{\Omega} \lambda \Delta \delta \theta \psi \mathrm{d} t \mathrm{~d} \Omega, \\
& =\mathcal{I}_{3}-\mathcal{I}_{4} \\
\mathcal{I}_{3} & =\int_{0}^{t_{f}} \int_{\Omega} \rho c \frac{\partial \delta \theta}{\partial t} \psi \mathrm{d} t \mathrm{~d} \Omega \\
& =\int_{\Omega}\left([\rho c \delta \theta \psi]_{0}^{t_{f}}-\int_{0}^{t_{f}} \rho c \delta \theta \frac{\partial \psi}{\partial t} \mathrm{~d} t\right) \mathrm{d} \Omega .
\end{aligned}
$$

Setting the initial condition $\delta \theta(x, y, z ; 0)=0$ in the sensitivity problem, we have

$$
\mathcal{I}_{3}=\int_{\Omega} \rho c \delta \theta \psi\left(. ; t_{f}\right) \mathrm{d} \Omega-\int_{0}^{t_{f}} \int_{\Omega} \rho c \delta \theta \frac{\partial \psi}{\partial t} \mathrm{~d} t \mathrm{~d} \Omega
$$

For the last term, we get

$$
\begin{aligned}
\mathcal{I}_{4}= & \int_{0}^{t_{f}} \int_{\Omega} \lambda \Delta \delta \theta \psi \mathrm{d} t \mathrm{~d} \Omega \\
= & \int_{0}^{t_{f}}\left(\int_{\partial \Omega} \lambda \frac{\partial \delta \theta}{\partial \vec{n}} \psi \mathrm{d} \delta \Omega-\int_{\partial \Omega} \lambda \delta \theta \frac{\partial \psi}{\partial \vec{n}} \mathrm{~d} \delta \Omega\right) \mathrm{d} t \\
& +\int_{0}^{t_{f}} \int_{\Omega} \lambda \delta \theta \Delta \psi \mathrm{d} \Omega \mathrm{d} t
\end{aligned}
$$
problem

Setting the boundary condition for the sensitivity

$$
\forall(x, y, z ; t) \in \partial \Omega \times T, \quad-\lambda\left(\frac{\partial \delta \theta}{\partial \vec{n}}\right)=h \delta \theta-\delta \Phi,
$$

we have

$$
\begin{aligned}
\mathcal{I}_{4}= & \int_{0}^{t_{f}} \int_{\partial \Omega}(-h \delta \theta+\delta \Phi) \psi \delta \Omega \mathrm{d} t \\
& -\int_{0}^{t_{f}} \int_{\partial \Omega} \lambda \delta \theta \frac{\partial \psi}{\partial \vec{n}} \mathrm{~d} \delta \Omega \mathrm{d} t \\
& +\int_{0}^{t_{f}} \int_{\Omega} \lambda \delta \theta \Delta \psi \mathrm{d} \Omega \mathrm{d} t \\
= & -\int_{0}^{t_{f}} \int_{\partial \Omega}\left(h \psi+\lambda \frac{\partial \psi}{\partial \vec{n}}\right) \delta \theta \delta \Omega \mathrm{d} t \\
& +\int_{0}^{t_{f}} \int_{\partial \Omega} \delta \Phi \psi \mathrm{d} \delta \Omega \mathrm{d} t+\int_{0}^{t_{f}} \int_{\Omega} \lambda \delta \theta \Delta \psi \mathrm{d} \Omega \mathrm{d} t .
\end{aligned}
$$

Consequently, the variation in the Lagrangian becomes

$$
\begin{aligned}
\delta \mathcal{L}= & \int_{0}^{t_{f}} \int_{\Omega} E \delta \theta \mathrm{d} t \mathrm{~d} \Omega \\
& +\int_{\Omega} \rho c \delta \theta \psi\left(. ; t_{f}\right) \mathrm{d} \Omega-\int_{0}^{t_{f}} \int_{\Omega} \rho c \delta \theta \frac{\partial \psi}{\partial t} \mathrm{~d} t \mathrm{~d} \Omega
\end{aligned}
$$




$$
\begin{aligned}
& +\int_{0}^{t_{f}} \int_{\partial \Omega}\left(h \psi+\lambda \frac{\partial \psi}{\partial \vec{n}}\right) \delta \theta \delta \Omega \mathrm{d} t \\
& -\int_{0}^{t_{f}} \int_{\partial \Omega} \delta \Phi \psi \mathrm{d} \delta \Omega \mathrm{d} t-\int_{0}^{t_{f}} \int_{\Omega} \lambda \delta \theta \Delta \psi \mathrm{d} \Omega \mathrm{d} t \\
& =\int_{0}^{t_{f}} \int_{\Omega}\left(E-\rho c \frac{\partial \psi}{\partial t}-\lambda \Delta \psi\right) \delta \theta \mathrm{d} t \mathrm{~d} \Omega \\
& +\int_{\Omega} \rho c \delta \theta \psi\left(. ; t_{f}\right) d \Omega-\int_{0}^{t_{f}} \int_{\partial \Omega} \delta \Phi \psi \mathrm{d} \delta \Omega \mathrm{d} t \\
& +\int_{0}^{t_{f}} \int_{\partial \Omega}\left(h \psi+\lambda \frac{\partial \psi}{\partial \vec{n}}\right) \delta \theta \delta \Omega \mathrm{d} t .
\end{aligned}
$$

As for Eqn. (7), the Lagrange multiplier $\psi$ has to be a solution of the following system of PDEs:

$$
\left\{\begin{array}{l}
\forall(x, y, z ; t) \in \Omega \times T, \\
\lambda\left(\frac{\partial^{2} \psi}{\partial x^{2}}+\frac{\partial^{2} \psi}{\partial y^{2}}+\frac{\partial^{2} \psi}{\partial z^{2}}\right)=E-\rho c \frac{\partial \psi}{\partial t}, \\
\forall(x, y, z) \in \Omega, \quad \psi\left(x, y, z ; t_{f}\right)=0, \\
\forall(x, y, z ; t) \in \partial \Omega \times T, \quad-\lambda\left(\frac{\partial \psi}{\partial \vec{n}}\right)=h \psi
\end{array}\right.
$$

Thus,

$$
\delta \mathcal{L}(\theta, I, \psi)=-\int_{0}^{t_{f}} \int_{\partial \Omega} \psi \delta \Phi \mathrm{d} t \mathrm{~d}(\partial \Omega) .
$$

Received: 11 February 2015 Revised: 31 August 2015

Re-revised: 27 February 2016 Accepted: 18 April 2016 